Julian Reif*

\title{
Touristische Aktionsräume und die Wahrnehmung von Crowding:
}

\section{Das Beispiel Tagestourismus in Hamburg}

DOI $10.1515 /$ tw-2019-0015

Zusammenfassung: Im Zuge der vielfältigen Diskussionen um eine touristische Übernutzung von Städten liegen kaum Untersuchungen über den Tagestourismus vor. Insbesondere über intradestinationale Aktionsräume und die damit in Verbindung stehende Wahrnehmung von anderen Menschen im Stadtraum gibt es im Gegenteil zu Studien über die Tourismusakzeptanz aufseiten der Einwohner wenig empirische Befunde aus touristischer Perspektive. Vor diesem Hintergrund ist es Ziel des Beitrages, Erkenntnisse über die Bewegungsmuster und die Wahrnehmung von anderen Stadtnutzern von Tagestouristen in Hamburg zu gewinnen. Dafür wurden die Aktionsräume mithilfe eines GPS-Trackers gemessen und durch einen Fragebogen die Wahrnehmung von Crowding ermittelt. Die Ergebnisse zeigen eine deutliche Konzentration des tagestouristischen Raum-Zeit-Konsums im Hamburger Central Business District. Andere Stadtnutzer werden zwar wahrgenommen, haben jedoch kaum Einfluss auf das aktionsräumliche Handeln vor Ort. Das positive Crowding überwiegt deutlich. Durch die Kombination von Aktionsräumen und der Wahrnehmung von Crowding können zwei Kernzonen in Hamburg identifiziert werden, die im Rahmen einer sozial verträglichen Tourismus- und Stadtentwicklung bevorzugt behandelt werden sollten.

Schlüsselwörter: GPS-Tracking, urbanes Crowding, touristische Aktionsräume, Städtetourismus, Tagestourismus

\begin{abstract}
In the course of the manifold discussions on tourist induced overuse of city destinations, there still is a lack of studies on same-day visitors. Additionally, in contrast to studies on the encounters between tourists and urban residents, so far there are only few empirical findings on intra-destination tourist movement patterns and the associated perception of urban crowds. Against this backdrop, this
\end{abstract}

\footnotetext{
*Corresponding author: Julian Reif: Institut für Management und Tourismus (IMT), Fachhochschule Westküste, Fritz-Thiedemann-Ring 20, D-25746 Heide, E-Mail: reif@fh-westkueste.de
} 
paper analyzes the spatio-temporal behavior and the perception of crowding of same-day visitors in Hamburg. The data collection process involves GPS tracking and a survey for estimating the perception of crowding. The results show a tourist space-time consumption that is clearly concentrated in Hamburg's Central Business District. Although other city users are perceived, they have hardly any influence on the time-space patterns. Furthermore, positive crowding clearly predominates. Combining the GPS based measured itineraries and the perception of crowding, two core zones in Hamburg are identified which should be given preferential treatment within the framework of sustainable tourism and urban development.

Keywords: GPS tracking, urban crowding, tourist movement patterns, urban tourism, same-day visitors

\section{$1 \quad$ Hintergrund und Zielsetzung}

In den letzten Jahren kam es bundesweit zu einem deutlichen Wachstum im Städtetourismus (u. a. Bauder, 2018). Das Anwachsen der urbanen Besucher stellt die städtische Infrastruktur, touristische Dienstleister und Einwohner gleichermaßen vor vielfältige Herausforderungen. Sowohl im medialen Diskurs als auch in der wissenschaftlichen Auseinandersetzung wird der räumlich und zeitlich auftretende Besucherandrang und die damit einhergehende Tourismus(in)akzeptanz der städtischen Gastgeber stark diskutiert (u. a. McKinsey \& Company \& WTTC, 2017; Postma \& Schmücker, 2017; Grube \& Novy, 2018; Koens, Postma \& Papp, 2018; Peeters et al., 2018). Dabei stehen in erster Linie die Touristenmetropolen Barcelona, Amsterdam, Venedig aber auch Dubrovnik und Berlin im Fokus der Betrachtung. Andere Städte, die durchaus ebenfalls eine beachtliche Bedeutung im (inter)nationalen Tourismus innehaben, werden kaum thematisiert. Es zeigt sich jedoch, dass auch der Blick auf Städte wie München oder Hamburg, die unter der „Spitze des Eisberges“ (Kagermeier \& Erdmenger, 2019) liegen, lohnt, um die Prozesse im Zusammenspiel zwischen Gastgeber, Touristen und Stadtraum zu verstehen. Der an touristisch frequentierten Attraktionspunkten der Städte auftretende Besucherdruck kann neben Belastungserscheinungen bei Einwohnern, auch das Erleben der Touristen beeinträchtigen. Allerdings wird sich der Frage, wie es um die soziale Tragfähigkeit der Gastgeber gestellt ist, deutlich ausführlicher gewidmet (bspw. Muler Gonzalez et al., 2018; Eusébio et al., 2018; Freytag \& Gomes de Matos, 2018). Eine Analyse der psychologischen bzw. perzeptuellen Tragfähigkeit aus Touristenperspektive - vor allem in Städten - wurde bislang nur in wenigen Studien untersucht (Popp, 2012, S. 50). Das Gästevolumen und 
das damit verbundene Wachstum spielt bei der Frage der Tourismusakzeptanz eine entscheidende Rolle (Peeters et al., 2018, S. 24). Daher ist es folgerichtig, sich dem in vielen Destinationen volumenstärksten touristischen Segment, dem Tagestourismus, in besonderer Weise $\mathrm{zu}$ widmen. Allerdings ist der touristische Teilbereich trotz seiner Bedeutung - im Vergleich zum Übernachtungstourismus - nur unzureichend untersucht (Flognfeldt jr., 2005, S. 54). Dies gilt für die Ermittlung sowohl von Umfang und Struktur als auch für die Ermittlung der raumzeitlichen Bewegungsmuster. Letzteres ist insofern beachtlich, da das Wissen, wie sich Touristen im Stadtraum bewegen, wie sie diesen wahrnehmen und erkunden, für eine nachhaltige Stadt- und Tourismusentwicklung wichtig ist (Edwards \& Griffin, 2013, S. 583). Durch die Analyse der raumzeitlichen Bewegungsmuster kann die touristische Infrastruktur optimiert, Besucherströme reguliert und negatives Crowding (vgl. Abschnitt 2.3) vermieden werden. Darüber hinaus kann das Wissen in der touristischen Produktentwicklung und zur Konzentration von Marketingbudgets eingesetzt werden, was wiederum zur Verbesserung des touristischen Vor-Ort-Erlebnisses und zur Verbesserung der Tourismusakzeptanz beitragen kann (Edwards et al., 2010, S. 104; Edwards \& Griffin, 2013, S. 582; Shoval \& Ahas, 2016, S. 589). Eine von Bauder bereits im Jahr 2012 postulierte Kombination digitaler Trackingtechnologien mit der Analyse soziokultureller Tragfähigkeit ist bislang ausgeblieben (Bauder, 2012, S. 428). In der Literatur finden sich mit Ausnahme der Arbeit von Bauder \& Freytag (2015) über „functional same-day-visitors“1 in Freiburg und der Analyse von Freytag (2010a) für Tagesbesucher in Heidelberg, zudem keine speziell auf das aktionsräumliche Verhalten von Tagestouristen abzielenden GPS-Analysen. Vor diesem Hintergrund ist es Ziel des Beitrages, Erkenntnisse über die Bewegungsmuster und die Wahrnehmung von anderen Stadtnutzern von Tagestouristen in Hamburg zu gewinnen. Konkret sollen dabei folgende Fragestellungen beantwortet werden:

- Welche Aktionsräume weisen Hamburger Tagestouristen auf und wo lassen sich Hotspots des Tagestourismus in Hamburg lokalisieren?

- Welche Rolle spielen andere Stadtnutzer beim Tagesausflug und hat dies Einfluss auf das aktionsräumliche Handeln von Touristen vor Ort?

- Wo im Stadtraum werden aus Sicht von Touristen viele Menschen wahrgenommen und werden diese als störend empfunden?

- Gibt es Besonderheiten in den Aktionsräumen von Tagestouristen, die Crowding verstärkt wahrnehmen?

1 „functional same-day visitors“: „[...] the total of overnight and same-day visitors who do not spend more than 24 hours (i.e. including a maximum of one overnight stay) at their destination [...]” (Bauder \& Freytag 2015, S. 686). 


\section{Theoretischer Rahmen}

\subsection{Touristische Aktionsräume und deren Einflussfaktoren}

Die Zeit, als begrenzte und begrenzende Ressource eines Menschen, kann als Nukleus der Erforschung von Aktionsräumen angesehen werden (Hägerstrand, 1970; Scheiner, 1998). Ausgehend vom Individuum wird bei der Weiterentwicklung klassisch geographischer Ansätze - wie dem der Zeitgeographie oder dem der Münchner Schule der Sozialgeographie - im Rahmen einer handlungstheoretischen Auslegung der Aktionsraumforschung der Aktionsraum einer Person durch sein Handeln konstituiert (Scheiner, 1998). Die so verstandene Erforschung von Aktionsräumen befasst sich jedoch fast ausnahmslos mit dem hochgradig routinisierten Alltagshandeln von Personen innerhalb eines „weekly movement cycle“ (Roseman, 1971, S. 590) ausgehend vom Wohnort. Im Gegensatz zu den alltäglichen Aktionsräumen, werden Urlaubs- und Geschäftsreisen als touristische Aktivitäten in der Aktionsraumforschung kaum berücksichtigt (Osterhage \&Wiegandt, 2014, S. 93). Dabei spielen im Tourismus Raum-Zeit-Bezüge eine wichtige Rolle. Touristische Angebote können bspw. als räumlich-zeitliche Gebilde (Steinbach, 2003, S. 1) bzw. der Tourismusmarkt als Handel von Raum-Zeit-Relationen begriffen werden (Wöhler, 2001, S. 190; Eisenstein, 2014, S. 6). Bei der Erforschung touristischer Aktionsräume wird der Blickwinkel auf das außeralltägliche Handeln von Personen gerichtet. Dabei lassen sich zwei Maßstabsebenen bestimmen: interregionale touristische Aktionsräume (im Sinne von QuellZielgebietsbeziehungen) sowie intraregionale touristische Aktionsräume (im Sinne eines aktionsräumlichen Verhaltens in der Destination) (McKercher \& Lau, 2008, S. 356). Interregionale oder auch interdestinationale touristische Bewegungsmuster gehen von einer zirkulär-räumlichen Mobilität als konstitutives Element des Tourismus aus (Leiper, 1979) und lassen sich mit dem Modellansatz der „tourist attraction systems“ (Leiper, 1990) näher erklären. Die touristischen Attraktionssysteme sorgen für divergierende zeiträumliche Bewegungsmuster und bestehen aus drei Elementen: Person mit touristischen Bedürfnissen (bspw. Shopping-Tagestourist), Nukleus (bspw. Kaufhaus in der Destination) und Marker (bspw. Information über die Öffnungszeiten des Kaufhauses). Da jede Person mit individuellen Reisemotiven und Informationen über zu bereisende Orte ausgestattet ist, entstehen in der Folge verschiedene zeiträumliche Muster (Schamel, 2017, S. 42). Im Zuge dessen lassen sich unterschiedliche Raummuster erklären und veranschaulichen: Eine dreiwöchige Rundreise im Westen der Vereinigten Staaten im Vergleich zu dem eher eingeschränkten Bewegungsprofil eines einwö- 
chigen Cluburlaubs auf Mallorca. Lau \& McKercher (2006, S. 41f) zeigen auf Basis verschiedener Studien insgesamt sechs unterschiedliche Kategorien interregionaler Bewegungsmuster von Touristen auf. ${ }^{2}$

Neben dieser Metaebene lassen sich auf kleinräumiger Ebene intraregionale bzw. intradestinationale touristische Aktionsräume in der Destination beobachten. Im Gegensatz zu den oben beschriebenen Aktionsräumen zwischen Destinationen wird sich der Untersuchung der Aktionsräume in der Destination kaum gewidmet (Leung et al., 2012, S. 471). Hinzu kommt, dass die Aktionsräume individuell sind und sich nach den persönlichen Interessen des Touristen richten (McKercher, 2004, S. 19). So lassen sich die Einflussfaktoren dieser individuellen Aktionsräume in zwei Hauptgruppen unterteilen: individuelle touristische sowie reisebezogene Faktoren (Shoval \& Isaacson, 2010, S. 15f). In Bezug auf die reisebezogenen Faktoren ist der Reiseanlass, bspw. Geschäfts- oder Urlaubsreise, einer der entscheidenden Gründe für die divergierende Ausgestaltung von Aktionsräumen. Zwar können sowohl der Geschäfts- als auch der Urlaubsgast dieselbe Infrastruktur zur Fortbewegung in einer Destination nutzen, aufgrund der vorherrschenden Motive des Geschäftstouristen (Eisenstein et al., 2019) wird er aber ein anderes zeiträumliches Verhalten in der Destination aufweisen als der Urlaubstourist, der sich in der Regel an den freizeittouristisch relevanten Sehenswürdigkeiten orientiert. Destinationserfahrung hat ebenfalls einen entscheidenden Einfluss auf das Mobilitätsverhalten vor Ort. So lässt sich zwischen Erst- und Wiederholungsbesuchern ein deutlich anderes Mobilitätsverhalten nachweisen (Freytag, 2010b, S. 55; Lau \& McKercher, 2006, S. 42 f.). Des Weiteren kann die Aufenthaltsdauer, als zeitliche Komponente, Einfluss auf das zeiträumliche Verhalten haben (Xiao-Ting \& Bi-Hu, 2012, S. 638f.), was sich im Falle von Tagestouristen durch die Absenz einer Unterkunft und die damit verbundene kürzere Aufenthaltsdauer nachvollziehbar veranschaulichen lässt. Eine der entscheidenden Einflussvariablen ist die Unterkunft (Shoval et al., 2011, S. 1608), während sozioökonomische Merkmale, die zu den oben genannten individuellen touristischen Faktoren zählen, geringe Auswirkungen auf unterschiedliche Raumaneignungsmuster haben (Shoval, 2002, S. 27). Neben diesen Einflussvariablen sind destinationsbezogene, externe Variablen relevant. Diese können - neben einem generellen Angebot an Sehenswürdigkeiten - „weiche Faktoren“ wie das Wetter oder die Anwesenheit von vielen Menschen sein, die u. U. dazu führen, spontan seine Pläne zu ändern (Shoval \& Isaacson, 2010, S. 18). Im Falle intradestinatio-

2 Die Kategorien lauten: „single point“, „,base site“, “stopover“, „,chaining loop“, „destination region loop“ und „complex neighborhood“ (Lau \& McKercher, 2006, S. 41; siehe dazu auch Oppermann 1995). 
naler Aktionsräume konnten bspw. McKercher \& Lau (2008) 78 Bewegungsmuster mit elf Bewegungsstilen bei einer Untersuchung in Hongkong nachweisen.

Ausgehend von den drei konstitutiven Elementen der „tourist attraction systems“ ergibt sich in Summe ein Modell des aktionsräumlichen Handelns von Touristen, welches, je nach dem zu betrachtenden Element des Systems, unterschiedliche abhängige Variablen aufweist, die einen aktionsräumlichen Handlungsvollzug des Touristen evozieren (Abb. 1). Diese Handlung im Raum intendiert oder nicht intendiert - hat Folgen für den Touristen selbst oder die Gesellschaft, und wiederum Einfluss auf die „tourist attraction systems“ und die verschiedenen abhängigen Variablen. Der touristische Aktionsraum wird so durch das Handeln der Touristen konstruiert und lässt sich durch die besuchten Orte in der Destination messen, die der Tourist „zur Befriedigung seiner mit dem Aufenthalt verbundenen Bedürfnisse und damit zur Erfüllung des Reisezwecks in Anspruch nimmt“ (Eisenstein, 2014, S. 15).

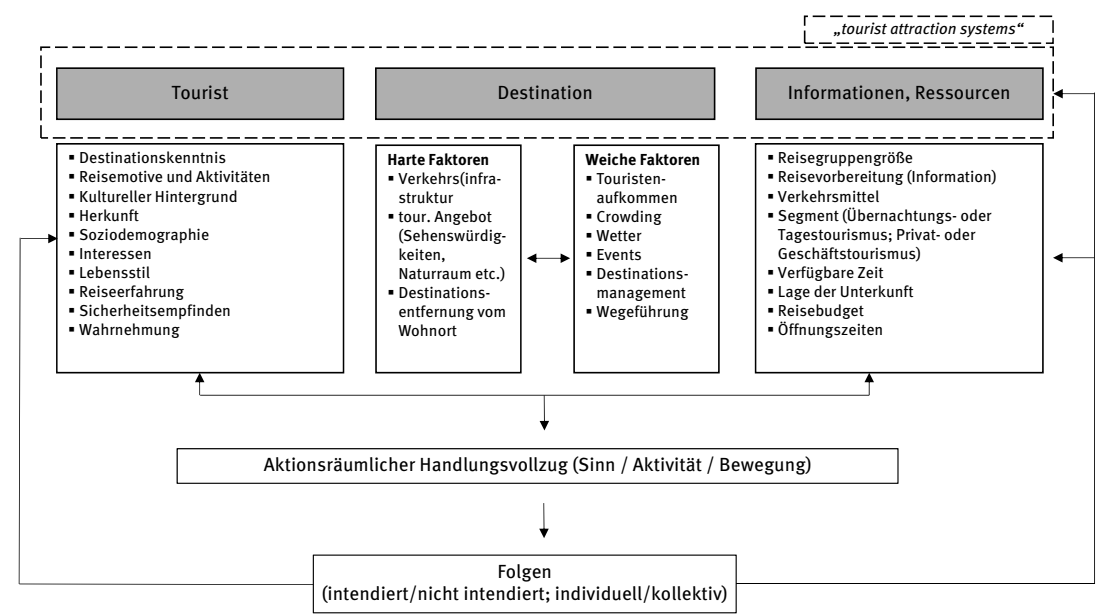

Abb. 1: Modell des aktionsräumlichen Handelns von Touristen. (Quelle: Eigene Darstellung auf Basis von Shoval \& Isaacson, 2010; Beeco \& Hallo, 2014; Lew \& McKercher, 2006; Schamel, 2017; Scheiner, 1998; 2000; Leiper, 1990; Steinbach, 2003.)

\subsection{Messung von touristischen Aktionsräumen}

Bei der Messung von touristischen Aktionsräumen kommen verschiedene analoge und digitale Messmethoden mit ihren spezifischen Vor- und Nachteilen zum Einsatz (u. a. McKercher \& Lau, 2009; Kellner \& Egger, 2016; Weber \& Bauder, 2013). In Bezug auf analoge Methoden sind hier vor allem Beobachtung (u. a. Keul \& Kühberger, 1996) und Befragung inkl. Tagesprotokolle (u. a. Becker, 1982; Debbage, 1991), also das Aufzeichnen sogenannter „time-space-budgets“ 
während eines Urlaubs, zu nennen. Bei den digitalen Trackingmethoden, die erstmals nach der Jahrtausendwende mit dem Einläuten eines digitalen Zeitalters des Touristentrackings in der Forschung eingesetzt wurden, ist das GPS-Tracking unabhängig davon ob via GPS-Tracker oder einem App-Tracking via Smartphone - die meist benutzte Methode, um das raumzeitliche Verhalten von Touristen zu messen (Shoval \& Ahas, 2016). Dabei bestehen vielfältige weitere digitale Möglichkeiten, raumzeitliche Bewegungsmuster von Touristen $\mathrm{zu}$ identifizieren. $\mathrm{Zu}$ nennen sind hier in erster Linie die Nutzung von passiven Mobilfunkdaten (u. a. Ahas et al., 2008; Reif, 2019) sowie Social Media und weitere Internetressourcen wie Nutzerdaten aus Online-Buchungsplattformen (u. a. Girardin et al., 2009; Batiste e Silva et al., 2018). Die Vorteile von GPS-Tracking liegen jedoch in der Genauigkeit in Bezug auf die räumliche und zeitliche Auflösung, der einfachen Bedien- und weltweiten Verfügbarkeit sowie in der automatisierten Integration in geographische Informationssysteme. Nachteilig ist, dass GPS-Tracking mit anderen Methoden kombiniert werden muss, um über eine rein visuell-deskriptive Analyse der zurückgelegten Wege hinauszukommen. Hohe Anschaffungskosten für das technische Equipment, die logistischen Herausforderungen der Aus- und Rückgabe der Trackingapplikation sowie der Feldzugang in GPS-Tracking-Studien stellen weitere einschränkende Faktoren dar (Schamel, 2017, S. 66; Shoval \& Isaacson, 2010, S. 82). Letzteres gestaltet sich insbesondere in Städten mit keinem eindeutigen Ein- oder Ausgang als äußerst schwierig (Shoval \& Isaacson, 2010, S. 98 f.). Gleichwohl findet das GPS-Tracking (in der nationalen wie internationalen Forschungslandschaft) verstärkten Einsatz und entsprechende Anwendung, u. a. mit verschiedenen Methodenkombinationen, in verschiedenen Destinationsarten aber auch bei unterschiedlichen Tourismussegmenten. ${ }^{3}$

\subsection{Urbanes Crowding: Die Perspektive der Touristen}

Urbaner Raum kann als sozial konstruiertes Produkt der Handlungen von Individuen (Touristen, Einwohner, Akteure etc.) verstanden werden und ist demnach einem ständigen Wandlungsprozess unterzogen (Massey, 2005, S. 9). In diesem Prozess der Raumkonstruktion nimmt der Tourist die Rolle eines Prosumers, als gleichzeitiger Produzent und Konsument von Raum bzw. dem touristischen Produkt, ein (Eisenstein, 2014, S. 106). Der Stadtraum wird folglich durch alle Raumnutzer produziert und Touristen sind ein bedeutender Teil davon (Pappale-

3 für eine Übersicht zur tourismusbezogenen GPS-Forschung sei u. a. auf Shoval und Ahas (2016, S. 561 ff.) und Reif (2019, S. 3) verwiesen. 
pore \& Smith, 2016, S. 97 f.). Das touristische Erlebnis bzw. die Qualität der Erfahrung in Bezug auf die Anwesenheit von anderen Touristen vor Ort wird anhand der Begegnungen mit anderen Raumnutzern sowie von den individuellen Aktivitäten und den Erwartungshaltungen der Touristen bestimmt (Pappalepore \& Smith, 2016, S. 97; Vester, 1993, S. 125). Folglich kann die Wahrnehmung von Crowding eine mögliche abhängige Variable mit Einfluss auf das aktionsräumliche Handeln von Touristen sein (Abb. 1).

Um sich der Wahrnehmung von Crowding zu nähern, muss das Konzept der „tourism carrying capacity“ (u. a. McCool \& Lime, 2001) näher betrachtet werden. Dabei unterscheiden Watson \& Kopachevsky (1996, S. 174 ff.) eine ökonomische, ökologische, physische, soziale und psychische Tragfähigkeit. Letztere ist dabei im vorliegenden Falle und in Abgrenzung zur sozialen Tragfähigkeit, bei der die Tourismusakzeptanz der Einwohner im Fokus steht, deshalb von Relevanz, da sie die Touristen selbst in den Blick nimmt (Tab. 1). Nach Martin \& Uysal (1990, S. 329) wird die psychische Tragfähigkeit überschritten, „when tourists are no longer comfortable in the destination area, for reasons that can include perceived negative attitudes of the locals, crowding of the area, or deterioration in the physical environment.” Als störend wahrgenommene Stadtnutzer überschreiten demnach die psychische Tragfähigkeitsgrenze (Neuts \& Nijkamp, 2012, S. 2135) und beeinflussen das touristische Erlebnis vor Ort. Die Grenzen der Tragfähigkeit sind sowohl bei der sozialen als auch bei der psychischen Tragfähigkeit im Vergleich bspw. zu einer ökologischen Tragfähigkeitsgrenze deutlich schwerer zu quantifizieren (Schmude \& Namberger, 2015, S. 105).

Tab. 1: Tourism Carrying Capacity im Städtetourismus (Quelle: Eigene Darstellung)

\begin{tabular}{ll}
\hline Art der Tragfähigkeit & Beispiel mit Bezug zum Städtetourismus \\
\hline ökonomische Tragfähigkeit & $\begin{array}{l}\text { Einwohner leiden unter zu hohen Miet- und Immobilienpreisen } \\
\text { durch tourismusinduzierte Gentrificationprozesse }\end{array}$ \\
\hline ökologische Tragfähigkeit & $\begin{array}{l}\text { Städtische Feinstaubbelastung durch tourismusinduzierten } \\
\text { PKW-Verkehr überschreitet festgeschriebene Grenzwerte }\end{array}$ \\
\hline physische Tragfähigkeit & $\begin{array}{l}\text { Fehlende bzw. ausgebuchte Beherbergungskapazitäten durch } \\
\text { städtisches Großevent }\end{array}$ \\
\hline soziale Tragfähigkeit & $\begin{array}{l}\text { Unzufriedenheit bei Einwohnern durch hohe touristische Akti- } \\
\text { vität in städtischen Wohnvierteln }\end{array}$ \\
\hline psychische Tragfähigkeit & $\begin{array}{l}\text { Verminderung der Gästezufriedenheit durch erhöhtes Touris- } \\
\text { tenaufkommen an innerstädtischen Sehenswürdigkeiten }\end{array}$ \\
\hline
\end{tabular}

Bei der Wahrnehmung von Crowding auf Seiten der Touristen muss zwischen einer objektiven physischen Dichte und der subjektiv-psychologischen Wahrnehmung dieser Dichte unterschieden werden (Pearce, 2011, S. 94). Die physische Dichte wird dabei als eine notwendige, nicht aber hinreichende Bedingung für 
die Wahrnehmung von Crowding angesehen (Stokols, 1972, S. 275). Die Wahrnehmung ist demnach eine psychologische Bewertung der Anzahl der Raumnutzer während des touristischen Erlebnisses (Shelby et al., 1989) oder anders ausgedrückt: Bei der Wahrnehmung von Crowding handelt es sich um ein sozio-psychisches Konstrukt (Neuts et al., 2012, S. 651). Drei Einflussfaktoren der Wahrnehmung von Crowding werden in der Forschung identifiziert: Situative Variablen (Anzahl von anderen Menschen etc.), soziale Variablen (bspw. Verhalten anderer Touristen) sowie persönliche, individuelle Variablen (bspw. Erwartungshaltung) (Neuts \& Vanneste, 2018, S. 404 f.) (Abb. 2).

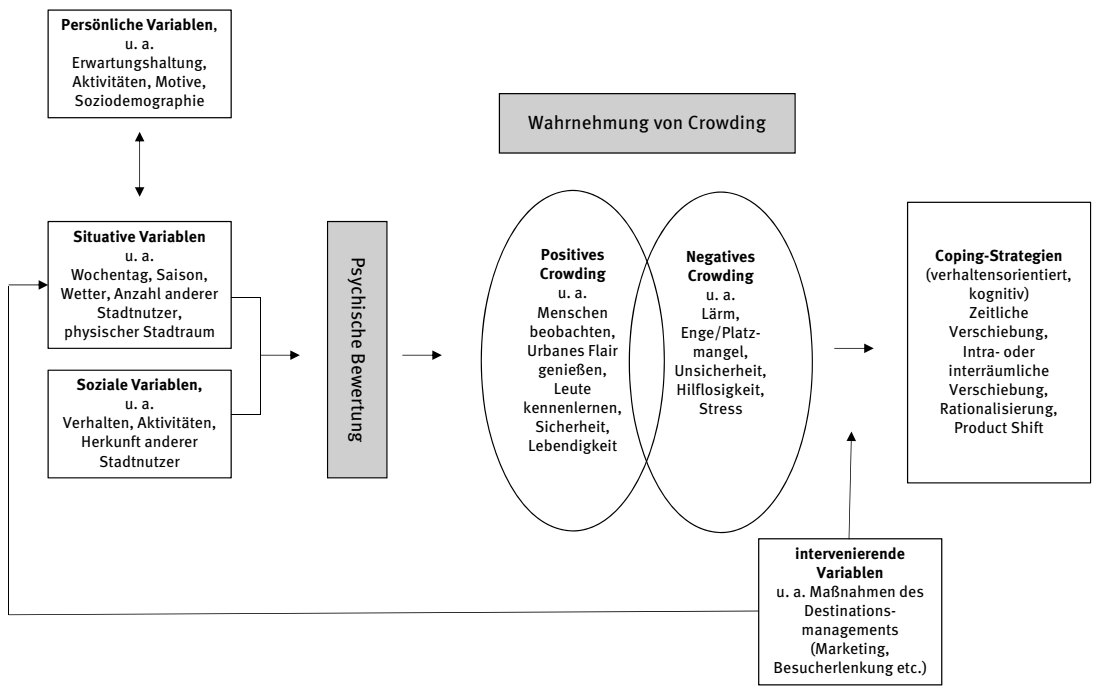

Abb. 2: Modell der Wahrnehmung von Crowding in urbanen Räumen. (Quelle: Eigene Darstellung auf Basis von Bryon \& Neuts, 2008; Popp, 2012; Schamel \& Job, 2014)

Allerdings ist die Frage, wie Touristen Menschenansammlungen wahrnehmen und wie sie mit ihnen umgehen, bislang in der städtetouristischen Forschung noch wenig behandelt worden (Popp, 2012, S. 50). Im Vergleich zu vielen Studien über Tourist-Tourist-Begegnungen und den Umgang mit der Wahrnehmung von vielen Menschen in Nationalparks, Wildnisgebieten und weiteren Outdoorsettings (u. v. a. Shelby et al., 1989; Vaske \& Shelby, 2008; Kalisch, 2012; Schamel \& Job, 2014; Zehrer \& Raich, 2016) wurde dem urbanen Raum bislang nur in einigen wenigen Crowding-Studien Beachtung geschenkt (Bryon \& Neuts, 2008; Jin \& Pearce, 2011; Popp, 2012; 2017; Neuts \& Nijkamp, 2012; Neuts et al., 2012; Neuts \& Vanneste, 2018). Dies hat auch damit zu tun, dass es schwierig - wenn nicht unmöglich - erscheint, für Städtebesucher zu erkennen, ob es sich bei der angetroffenen „urbanen Crowd“ um Touristen oder weitere raumwirksame Stadtnutzer handelt. Was in anderen touristischen Segmenten, bspw. bei Betrachtung 
des Tourismus in Naturräumen zumeist kein Problem darstellt, gestaltet sich im städtischen Raum deutlich komplexer und wird als eine der Schwierigkeiten beim Übertrag der Erkenntnisse aus der Crowdingforschung in einen städtischen Kontext gesehen (Popp, 2012, S. 53). Weitere Besonderheiten im städtischen Raum bestehen in der Multioptionalität der Städte und der Tatsache, dass es auch ein sogenanntes „good crowding“ (Wickham \& Kerstetter, 2000, S. 169) geben kann (Popp, 2012, S. 53). Good crowding oder positives Crowding kann als psychologische Interpretation beschrieben werden, die auftritt, wenn Touristen vielen anderen Menschen im Raum begegnen und sie die Vor- und Nachteile zugunsten der Vorteile abwägen (Sun \& Budruk, 2017, S. 1260). Das positive Empfinden der Anwesenheit von vielen Menschen ist dahingehend ein interessantes Phänomen, da das Gegenteil, eine Menschenleere, einem insbesondere im urbanen Kontext als befremdlich vorkommen könnte. Eine menschenleere Fußgängerzone an einem Samstagmittag würde aller Voraussicht nach ein unbehagliches Gefühl auslösen, wohingegen ein ähnliches Setting an einem Strand eher kein Unbehagen hervorrufen würde. In lebendigen Innenstädten hingegen kann somit von einem „collective gaze“ (Urry, 2002, S. 150) gesprochen werden, der dafür sorgt, dass dort, wo viele Menschen anzutreffen sind, offenbar „etwas los ist“ und man am „richtigen Platz“ ist (Popp, 2017, S. 361).

Für die Wahrnehmung von Crowding im Kontext des Städtetourismus lassen sich schließlich folgende Kernpunkte festhalten:

- Persönliche, situative und soziale Variablen beeinflussen die Wahrnehmung von Crowding.

- Menschenansammlungen im städtischen Raum müssen nicht als negativ angesehen werden bzw. die negative Konnotation von Crowding muss im urbanen Kontext infrage gestellt werden (Neuts \& Nijkamp, 2012, S. 2148).

- Der Grat zwischen der Wahrnehmung von positivem und negativem Crowding ist schmal (Popp, 2012, S. 63).

- Durch entsprechende Anpassungsstrategien wie bspw. eine zeitliche und räumliche Verschiebung des Aufenthaltes, lassen sich die negativen Begleitumstände abmildern (Popp, 2012).

- Das Destinationsmanagement kann durch Maßnahmen Einfluss auf situative Variablen und mögliche Anpassungsstrategien nehmen (bspw. durch Besucherlenkung). 


\section{$3 \quad$ Methodik}

\subsection{Der Untersuchungsraum Hamburg}

Die Stadt Hamburg zeichnet sich nicht zuletzt mit der Elbphilharmonie als neues cultural landmark als eine beliebte Touristenmetropole aus, die insbesondere in den letzten zehn Jahren ein deutlich überdurchschnittliches Wachstum vorweist ${ }^{4}$ und die im Jahr, 2017 mit 13,8 Mio. amtlich erfassten Übernachtungen in Beherbergungsbetrieben ab zehn Betten nach Berlin und München die Stadt mit der bundesweit dritthöchsten Übernachtungszahl war (Statistisches Bundesamt, 2018). Zusätzlich zu den in der Statistik erfassten Übernachtungen finden jährlich über 7 Mio. Übernachtungen bei Verwandten und Bekannten, bei Privatvermietern und auf Campingplätzen statt. ${ }^{5}$ Neben dem Übernachtungstourismus ist Hamburg ein beliebtes Tagesreiseziel. Auf eine Übernachtung entfallen nach oben genannten Angaben ca. vier inländische Tagesreisen und an einem Durchschnittstag bevölkern ca. 230.000 Tagestouristen die Stadt (Maschke, 2014, S. 2). Der Tagestourismus trägt darüber hinaus zu 51 \% des touristischen Gesamtkonsums im Jahr 2015 bei (Becker et al., 2019, S. 6) und ist damit nach den Kriterien Besuchervolumen und touristischer Konsum das bedeutendste Segment im Hamburger Städtetourismus.

Trotz der hohen touristischen Bedeutung zeigen Studien zur Tourismusakzeptanz, dass sich $94 \%$ der Hamburger nicht vom Tourismus gestört fühlen. Nur in den touristisch stark frequentierten Stadtteilen kommt es hingegen bereits $\mathrm{zu}$ ersten Anzeichen von antitouristischen Stimmungen. So fühlen sich $16 \%$ der Hamburger Einwohner in den Stadtteilen St. Pauli, Hamburg Altstadt und Neustadt, St. Georg, Hafencity und Sternschanze persönlich durch Touristen in Hamburg gestört bzw. beim Besuch von Veranstaltungen oder der Nutzung von anderen Angeboten durch die Touristen beeinträchtigt (Lorkowski, 2017, S. 7). Störfaktoren sind insbesondere die Überfüllung von Innenstadt und Restaurants, der Verkehr und Lärm. Gleichwohl ist in Hamburg die derzeitige Entwicklung der Tourismusakzeptanz nicht als ein akutes Problem anzusehen (Lorkowski, 2017; Beyer et al., 2017; Wybraniec, 2018). Die Quartiere mit einem hohen Besucherdruck scheinen weniger problematisch wahrgenommen zu werden als bspw. in

\footnotetext{
4 Zugewinn an amtlich erfassten Übernachtungen in Beherbergungsbetrieben im Zeitraum von 2007 bis 2017 in Höhe von +87\% (Bundesgebiet: +27\%) (Eigene Berechnung auf Basis Statistisches Bundesamt, 2008; 2018).

5 Bezugsjahr 2014; Camping: Touristik- und Dauercamping, Reisemobilisten (HHT \& DWIF, 2015, S. 8).
} 
anderen Städten und sowohl aus Experten- als auch aus Einwohnersicht ist die Verteilung des Übernachtungsangebotes kein bzw. ein geringes Problem (Beyer et al., 2017, S. 34). Ein akuter Handlungsbedarf - insbesondere im Bereich der Sharing-Economy und den damit einhergehenden Problemen (Stors \& Kagermeier, 2017) - ist derzeit nicht erkennbar. Gleichwohl sollte sich frühzeitig auf mögliche zukünftige Entwicklungen eingestellt und vor allem das teilweise problematische und konfliktträchtige Verhalten von Besuchern im Rahmen von Großveranstaltungen (Schlagermove, Harley Days) und auch im Kiez um die Reeperbahn beobachtet werden (Beyer et al., 2017, S. 34). Über das Empfinden der Touristen liegen in Bezug auf die Wahrnehmung des Tourismus, dessen Bewertung und zu den raumzeitlichen Bewegungsmustern jedoch keine Daten vor.

\subsection{Erhebungsdesign}

Um die vorliegenden Fragestellungen $\mathrm{zu}$ beantworten, wurden in zwei Erhebungswellen Tagestouristen bei ihrem Tagesausflug nach Hamburg getrackt und mithilfe eines Fragebogens über ihre Wahrnehmung von Crowding befragt. Im Herbst 2017 wurden auf Basis einer willkürlichen Auswahl mithilfe eines nach-

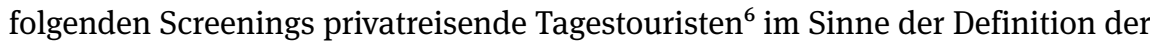
Welttourismusorganisation (UNWTO) (UNSD \& UNWTO, 2010, S. 10) an touristisch relevanten Orten in Hamburg angesprochen (u. a. Elbphilharmonie). Die Stichprobe berücksichtigt nur den Incoming-Tagestourismus sowie den sekundären Tagesausflugsverkehr, der durch Tagesausflüge vom Urlaubsort aus nach Hamburg entsteht. Der Binnentagestourismus der Hamburger Einwohner ist nicht in der Stichprobe enthalten. Den so identifizierten Tagestouristen wurden GPS-Tracker (QStarz BT-Q1000XT) ausgehändigt, deren Signalsetzung auf fünf Sekunden eingestellt wurde, um einen guten Mittelweg zwischen Gehgeschwindigkeit, Positionsbestimmung und Datenmenge zu erhalten (Chatel-Messer, 2013, S. 88). Gleichzeitig wurde ein Fragebogen verteilt, dessen Daten mithilfe einer einheitlicher ID anonymisiert mit dem GPS-Track verknüpft wurden. Fragebogen und GPS-Tracker konnten mit einem vorfrankierten Rückumschlag im Nachgang des Tagesausflugs an den Autor verschickt werden. Für die elf Erhebungstage standen insgesamt 40 GPS-Tracker zur Verfügung, die an Samstagen sowie an Sonn- und Feiertagen an die Touristen ausgehändigt wurden. In der zweiten

6 Geschäftsreisende Tagesgäste wurden angesichts deren knappen Zeitbudgets und aufgrund des Besuchs von Geschäftspartnern vermutlich stark eingeschränkten Bewegungsradius bewusst außen vorgelassen. 
Erhebungswelle, im Herbst 2018, wurden ergänzend dazu, Tagestouristen auf dem Weg nach Hamburg auf ihrer Fahrt im Regionalexpress zwischen Heide und Hamburg-Altona willkürlich im Zug angesprochen, da sich die Ansprache im Stadtraum aufgrund der Wetterverhältnisse und des oft knappen Zeitbudgets als schwierig darstellte. In Summe ergaben sich somit 163 befragte Tagestouristen, von denen 126 ein GPS-Track vorweisen konnten. Die GPS-Tracks wurden ausgelesen, mit der entsprechenden ID des Befragten versehen und zunächst in das Programm TibcoSpotfire eingelesen. Neben einer visuellen Plausibilitätsprüfung erfolgte die Bereinigung der Stichprobe in Anlehnung an Schuessler \& Axhausen (2008) sowie an Beeco et al. (2013). GPS-Punkte mit in Bezug auf Hamburg widersprüchlichen Höhen- sowie Längen- und Breitenangaben als auch unrealistische Geschwindigkeitsangaben wurden entnommen. Letzten Endes wurden nur die Trackpoints berücksichtigt, die innerhalb der amtlichen Gemeindegrenzen der Stadt Hamburg lagen. In der Summe ergaben sich von den 126 GPS-Tracks 96.412 Punkte innerhalb der Stadtgrenzen Hamburgs, die analysiert werden konnten. Mittels einer einheitlichen ID wurden die SPSS-Daten mit den GPS-Geokoordinaten verknüpft und sowohl in TibcoSpotfire als auch in ArcGis angefiltert und analysiert (u. a. Weber \& Bauder, 2013, S. 104 f). Der Vorteil liegt dabei in der Darstellung der Trackpoints nach verschiedenen Merkmalsausprägungen (bspw. Alter, Crowdingbetroffenheit, Aktivitäten usw.).

\subsection{Methodische Limitationen}

Die nachfolgenden Ergebnisse weisen einige Limitation auf, die bei der Interpretation beachtet werden müssen. Zunächst ist $\mathrm{zu}$ nennen, dass die vorliegende Stichprobe nicht repräsentativ für die Grundgesamtheit der Incoming-Tagestouristen ist, sodass die Übertragung der Ergebnisse auf die Gesamtheit aller Tagestouristen, die nach Hamburg reisen, nicht ohne weiteres möglich ist. Vielmehr handelt es sich im vorliegenden Falle um eine Convenience-Stichprobe. Zwar erfolgte die Stichprobenziehung durch eine willkürliche Ansprache mit darauffolgendem Screening, durch die Untersuchungsanlage der Befragung (u. a. im Zug) ist zumindest hinsichtlich der Herkunft der Tagesausflügler eine Schiefe zugunsten von Schleswig-Holstein in den Daten enthalten. ${ }^{7}$ Des Weiteren ist durch die Anlage des Feldzugangs im Zug zu berücksichtigen, dass der Bahnhof Altona, als ein wesentlicher Zugang zur Stadt, in den Hotspots zu relativieren ist,

7 Dennoch ist Schleswig-Holstein der Hauptquellmarkt für private und geschäftliche Tagesreisen nach Hamburg (41 \%) (Maschke, 2014, S. 5). 
da er sowohl auf der Hin- als auch auf der Rückreise in den GPS-Daten auftritt. Ein ähnliches Problem ergibt sich bei GPS-Trackingstudien mit Touristen, die den GPS-Tracker an einem bestimmten Ort zurückgeben müssen und dadurch vermehrt Rundwege entstehen. Des Weiteren ist zu berücksichtigen, dass nicht alle Reisenden ihren gesamten Tagesausflug über getrackt wurden. Dies hat mehrere Gründe: Neben der Tatsache, dass Touristen während ihres Tagesausflugs angesprochen wurden und damit der Ausflug in Teilen bereits vorüber war, sorgen GPS-Signalausfälle sowie die Datenbereinigung dafür, dass in den einzelnen Tracks Lücken vorhanden sind. Sicherlich ist zu guter Letzt nicht auszuschließen, dass sich das aktionsräumliche Verhalten in den Sommermonaten anders gestaltet und bspw. die Stadtstrände entlang der Elbe oder die Außenalster vermehrt frequentiert werden.

\section{Präsentation und Diskussion der Ergebnisse}

\subsection{Charakteristik der Stichprobe}

Zunächst erfolgt eine kurze Beschreibung der Stichprobe der Tagestouristen. Von den insgesamt 163 Tagesausflüglern sind $61 \%$ weiblich und $39 \%$ männlich. Das Durchschnittsalter beträgt 40,5 Jahre, bei einer relativen Gleichverteilung der Altersklassen (jünger als 26 Jahre: 22 \%; 26 bis 35: $22 \%$; 35 bis 45: $18 \%$; 46 bis 55: $19 \%$; älter als 55: $20 \%$ ). Schleswig-Holstein ist der Hauptquellmarkt (60\%), gefolgt von Niedersachsen und Nordrhein-Westfalen (jeweils $13 \%$ ) sowie privaten Tagestouristen aus dem restlichen Bundesgebiet und dem angrenzenden Ausland (14\%). Knapp zwei Drittel (61 \%) gaben an, 1 bis 3 Tagesausflüge pro Jahr nach Hamburg zu unternehmen, der Rest der Tagesausflügler unternimmt 4 und mehr Tagesausflüge pro Jahr in die Hansestadt. In Bezug auf die Aktivitäten stellte sich der Restaurantbesuch (70 \%) als sehr wichtige bzw. wichtige Aktivität beim Tagesausflug nach Hamburg heraus, gefolgt von spazieren gehen (44\%), Shopping (39\%) und Sightseeing (37\%). Spezielle Aktivitäten wie der Besuch von Sportveranstaltungen (7\%), Kinobesuch (6\%) oder Radfahren (3\%) standen hingegen nicht so stark im Fokus der Tagesausflügler. 


\subsection{Touristische Aktionsräume in Hamburg}

\subsubsection{Visuelle Trackanalyse}

Neben einer visuellen Interpretation der erhobenen GPS-Trackpoints erfolgt in den nachfolgenden Abschnitten eine Berechnung statistisch signifikanter Hotspots im Hamburger Tagestourismus. Ziel ist die Identifikation der tagestouristischen Aktionsräume von Besuchern in Hamburg.

Eine rein visuell-deskriptive Analyse der GPS-Trackpoints (Abb. 3) zeigt neben der deutlichen Dominanz von Trackpoints im Bezirk Hamburg-Mitte vier Hotspots des Hamburger Tagestourismus:

- der Bereich um den Hauptbahnhof, entlang der Spitaler- und Mönckebergstraße bis hin zum Jungfernstieg,

- eine „Ameisenstraße“ entlang der Elbe von den Landungsbrücken über den Baumwall/Elbphilharmonie hin zur Speicherstadt/HafenCity

- das Areal um die Reeperbahn in St. Pauli sowie

- den Bahnhof Altona.
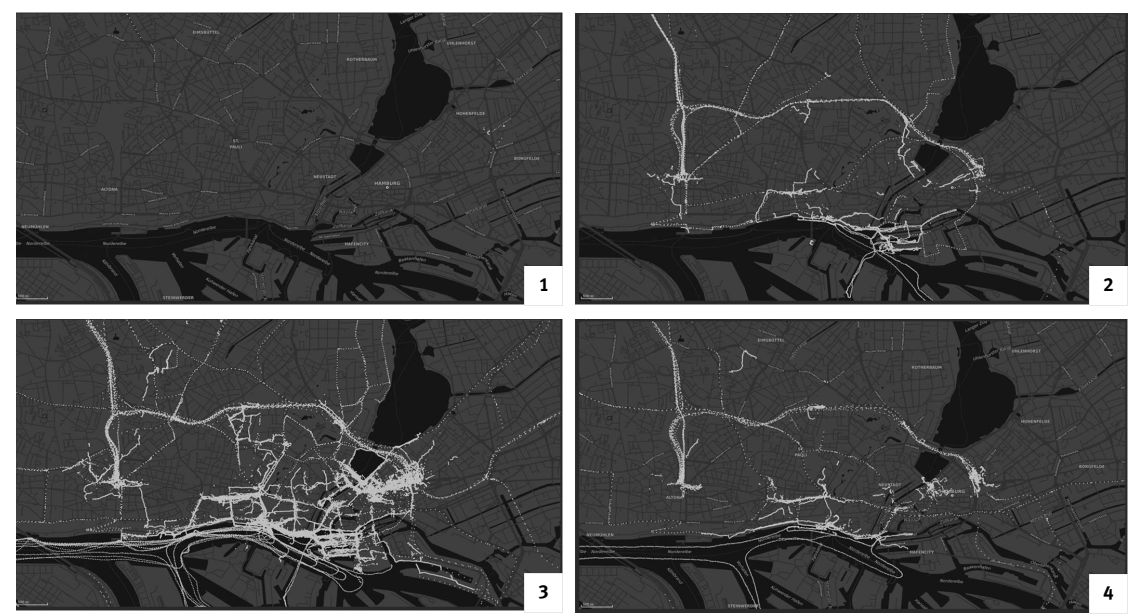

Abb. 3: GPS-Trackpoints von Hamburger Tagesausflüglern nach Tageszeiten. 1: 0:00 bis 05:59 Uhr, $\mathrm{n}=126$ Trackpoints; 2: 06:00 bis 11:59, $\mathrm{n}=309$ Trackpoints; 3: 12:00 bis 17:59 Uhr, $\mathrm{n}=66.068$ Trackpoints; 4: 18:00 bis 23:59 Uhr, $\mathrm{n}=909$ Trackpoints (Quelle: Eigene Erhebung, Berechnung und Darstellung. Kartengrundlage: TibcoSpotfire)

Gut zu erkennen sind ebenfalls die Routen der Hafenrundfahrten sowie die Straßenbahnlinien zwischen dem Bahnhof Altona und dem Hauptbahnhof sowie vereinzelt aufgesuchte Stadträume außerhalb des Zentrums (so zum Beispiel das 
Volksparkstadion im Nordwesten der Stadt). Bei der Aufteilung nach Tageszeiten zeigt sich eine zeiträumliche Ballung des Tagestourismus. Während in den Morgenund Abendstunden kaum Trackpoints vorhanden waren, waren Tagestouristen vor allem im Zeitraum zwischen 12:00 und 18:00 Uhr in Hamburg anzutreffen.

\subsubsection{Tagestouristischer Raumzeitkonsum}

Zur Objektivierung der Daten wird ein Netz mit einer Zellgröße von 100 x 100 m über die Stadt gelegt und über eine Join-Funktion die ermittelten GPS-Trackpoints in den Netzpolygonen gezählt. Durch die fünfsekündliche Aufzeichnung des GPSTrackers ist die Anzahl der Punkte je Hektar gleichzusetzen mit der verbrachten Zeit in einer Zelle (Bauder \& Freytag, 2015, S. 688). Das Ergebnis ist daher der tagestouristische Zeitkonsum in der Stadt je Hektar (Abb. 4). Die Zelle mit dem höchsten Zeitkonsum befindet sich in St. Pauli. Auch hier zeigen sich nochmal deutlicher die bereits in der visuellen Analyse ermittelten Ballungsräume. Gleichsam lassen sich so auch die "tagestouristischen Nicht-Orte“ oder „Not Spots“ (Shoval \& Isaacson, 2010, S. 160) des Hamburger Tagestourismus ermitteln. Zumindest in der vorliegenden Stichprobe dient der Hauptbahnhof als physische

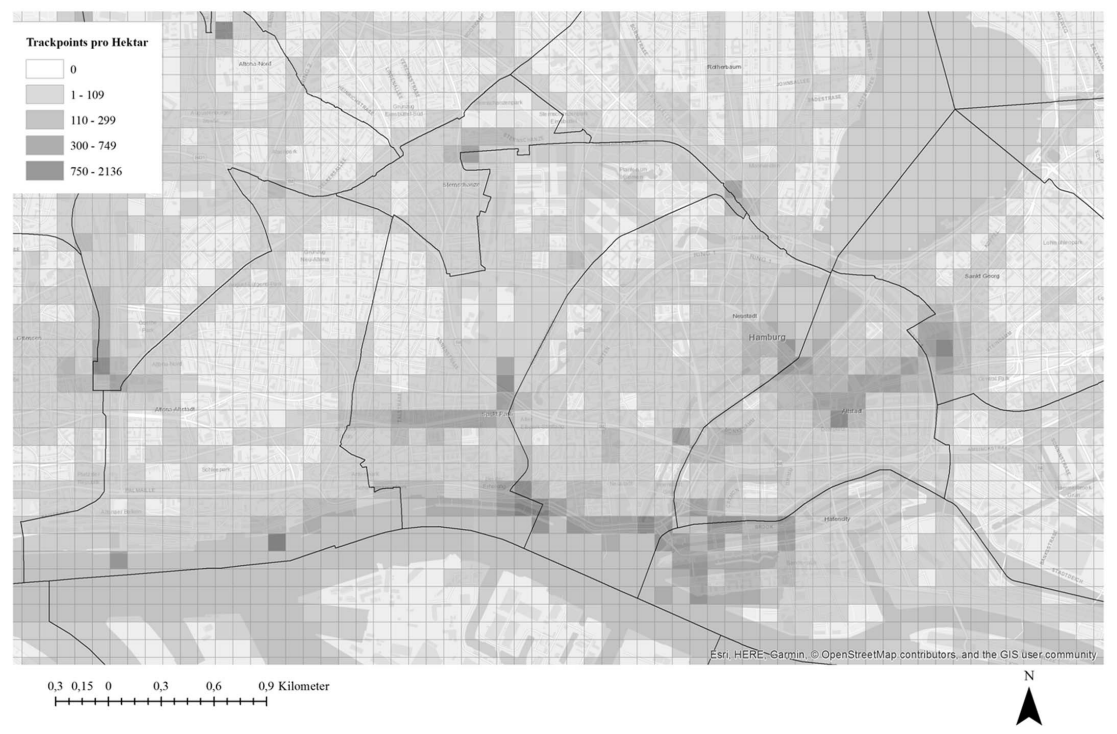

Abb. 4: Touristischer Raum-Zeitkonsum von Tagesausflüglern im Zentrum von Hamburg. $\mathrm{n}=126$ Tracks. Netzweite: $100 \times 100$ m. Klassengrenzen: Natürliche Unterbrechungen. (Quelle: Eigene Erhebung, Berechnung und Darstellung. Kartengrundlage: ALKIS Hamburg, ArcGis und OpenStreetMap) 
Barriere im Raum, da der Stadtteil St. Georg nordöstlich vom Bahnhof nahezu keine touristische Aktivität aufweist. Ähnlich auffallend ist, dass die Außenalster wenig frequentiert ist und die Kieze Schanzen- und Karolinenviertel im Vergleich zur Innenstadt deutlich weniger touristische Aktivität aufweisen. Die sich so gut wie frei von touristischer Aktivität auszeichnenden Kieze in Altona und Ottensen, aber auch die Außenbezirke Hamburgs sind - bis auf wenige Ausnahmen - nicht besucht worden.

Zusammenfassend lässt sich in Bezug auf die tagestouristischen Aktionsräume festhalten, dass eine durch das Verlassen der sogenannten „tourist bubble“ (Judd, 1999) veränderte Raumaneignung im Städtetourismus (Kagermeier, 2015, S. 211f) nicht stattfindet. Das Eindringen der Touristen in „new tourism areas“ (Maitland \& Newman, 2004) wie bspw. in die Hamburger Wohnviertel im Sinne eines „new urban tourism“ (Füller \& Michel, 2014, S. 1305 f.) lässt sich zumindest für den Hamburger Tagestourismus nicht beobachten. ${ }^{8}$ So kommt es in der Stadt demnach nicht zu einem „bottom-up“ getriebenen Touristifizierungsprozess (Freytag \& Bauder, 2018) durch Tagestouristen, sondern vielmehr zu einem überproportionalen touristischen Raum- und Zeitkonsum im innerstädtischen Central Business District (CBD) und damit dem klassisch-touristischen Zentrum. Die Folge ist ein Zentrum-Peripherie-Gefälle des Hamburger Tagestourismus, welches sich an der verzerrten Darstellung des touristischen Raum-Zeit-Konsums mittels Chronomap (Pohl, 2010) deutlich ablesen lässt (Abb. 5, rechts). Bei dieser Darstellung wird die Topologie der Hamburger Stadtteile aufrechterhalten, die Topographie hingegen verworfen und die jeweiligen Stadtteilpolygone werden proportional zur verbrachten Zeit je Stadtteil ausgewiesen.

\subsubsection{Hotspots des Hamburger Tagestourismus}

Bei der Analyse von Trackpoints stellt sich die Frage, ob die Punkte zufällig im Raum verteilt sind oder einem bestimmten Muster folgen und die gefundenen Punkthäufungen statistisch signifikant sind. In Ergänzung zur oben durchgeführten visuellen Trackanalyse kommt zur Ermittlung statistisch signifikanter Hotspots des Hamburger Tagestourismus daher eine Getis-Ord Gi^ Statistik zum Einsatz, wie sie im Spatial Statistic Tool von ArcGis „Optimierte Hot-Spot-Analyse“

\footnotetext{
8 Dies mag sich bei Vorhandensein einer Unterkunft, als einem entscheidenden Einflussfaktor des aktionsräumlichen Verhaltens, für den hamburgischen Übernachtungstourismus sicherlich anders gestalten und könnte ebenfalls mithilfe von GPS-Tracking, bspw. anhand von Übernachtungstouristen in über Plattformen gebuchten Privatwohnungen, untersucht werden.
} 


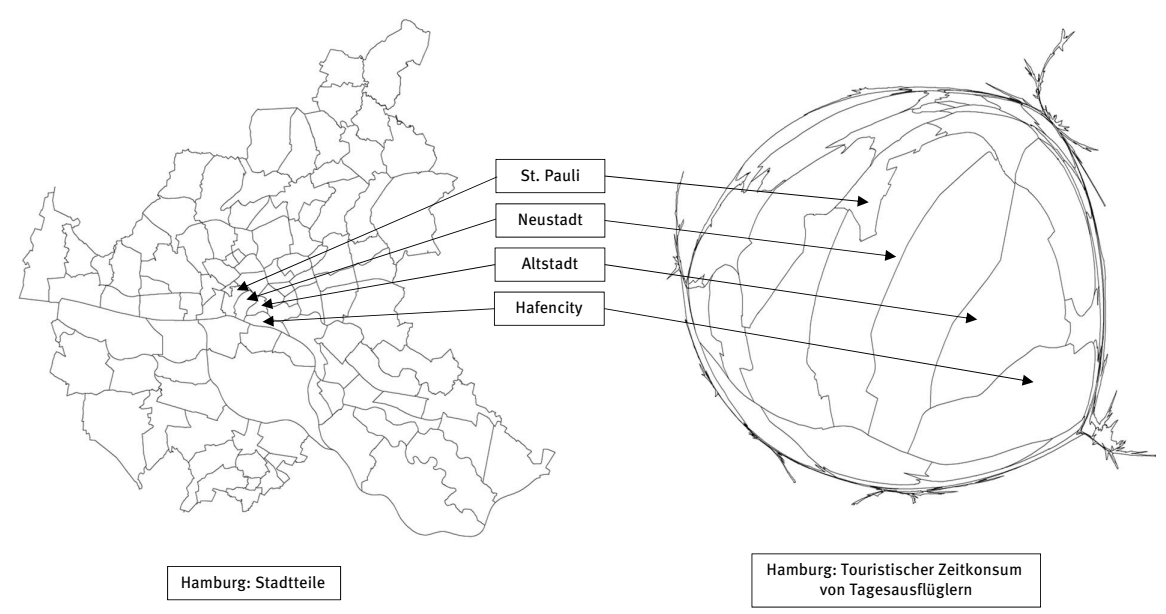

Abb. 5: Chronomap: Touristischer Raum-Zeitkonsum von Tagesausflüglern in Hamburg. $n=126$ GPS-Tracks. Analyse: Flächenkartogramm nach Gastner \& Newman („Cartogram“). (Quelle: Eigene Erhebung, Berechnung. Darstellung nach Pohl, 2010, S. 168. Kartengrundlage: ALKIS Hamburg)

integriert ist. Das Tool berechnet „statistisch signifikante räumliche Cluster mit hohen Werten (Hot-Spots) und mit niedrigen Werten (Cold-Spots)“ (ESRI, 2018, o. S.). Dabei wird eine Nullhypothese (alle Werte sind räumlich voneinander unabhängig) aufgestellt und für jedes Feature (GPS-Trackpoint) eine neue Ausgabe-Feature-Class mit einem p-Wert (Wahrscheinlichkeit), z-Wert (Standardabweichung) und Gi_Bin-Wert (Konfidenzniveau) kalkuliert (ESRI, 2018, o. S.). Im vorliegenden Beispiel kommt die Analysemethode „snap nearby incidents to create weighted points“ zum Tragen, die für den Einsatz bei vielen lagegleichen Punkten geeignet ist, um signifikante Aussagen über das räumliche Muster zu treffen (ESRI, 2018, o.S.). Dabei wird eine Fangentfernung berechnet und zum Aggregieren nahe gelegene GPS-Trackpoints verwendet. Jedem so aggregierten neuen gewichteten Punkt wird eine Anzahl zugewiesen, welche die Anzahl der zusammen gefangenen GPS-Trackpoints widerspiegelt. Im Anschluss erfolgt für eine bessere Darstellung und Lesbarkeit der Ergebnisse eine Interpolation der Ergebnisse anhand der Gi_Bin-Werte. Dazu kommt das Werkzeug „IDW (Inverse Distance Weighted)“ in der ArcGis-Toolbox „Geostatistical Analyst“ zum Einsatz. Das Ergebnis zeigt die statistisch signifikanten Hotspots anhand der GPS-Trackpoints der Tagestouristen in Hamburg unterschieden nach Konfidenzniveau als Rasteransicht. Die dunkelroten Bereiche in Abb. 6 mit einem Gi_Bin-Wert größer 2,624 zeigen demnach die tagestouristischen Hotspots mit einem $99 \%$-igen Konfidenzniveau.

Bei der Interpretation der Ergebnisse zeigt sich, dass sich einige Hotspots dadurch ergeben, dass sich ein einzelner Tagestourist lange an einem Ort auf- 


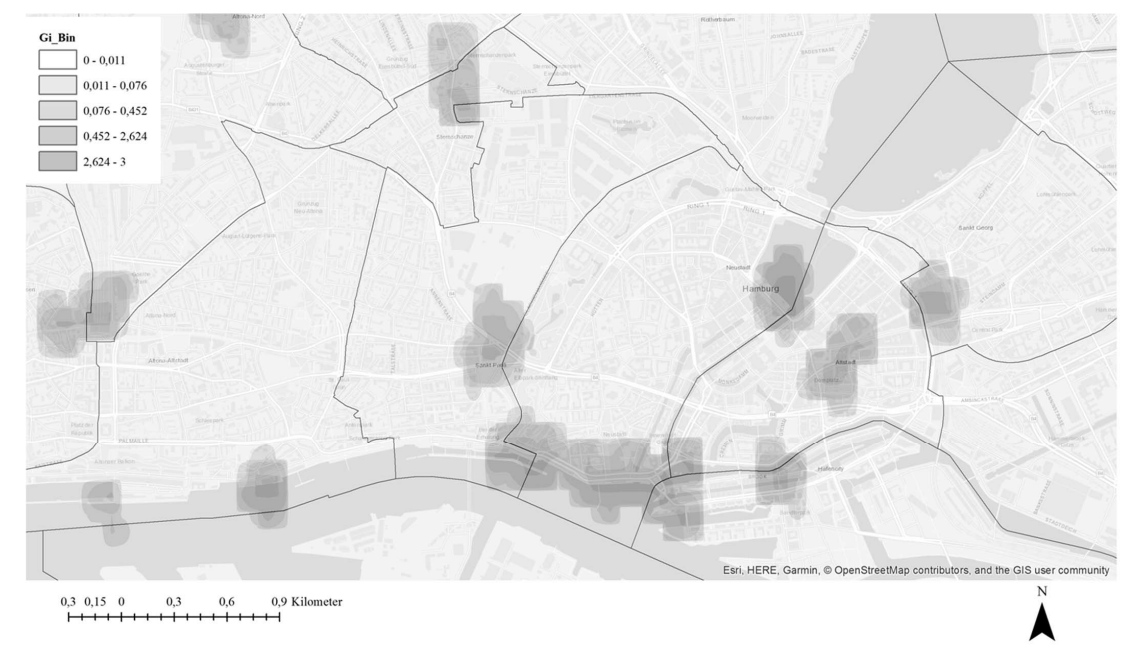

Abb. 6: Statistisch signifikante Hotspots des Tagestourismus in Hamburg. $n=126$ GPS-Tracks. (Quelle: Eigene Erhebung, Berechnung und Darstellung. Kartengrundlage: ALKIS Hamburg, ArcGis und OpenStreetMap)

gehalten hat. So bspw. ein Hotspot in Altona-Nord oder auch der Hotspot an der Fischauktionshalle direkt an der Elbe. Die anderen auf der Karte visualisierten signifikanten Hotspots werden hingegen durch viele einzelne Tagestouristen herbeigeführt. Zu nennen sind hier von West nach Ost:

- die Areale um die beiden S-Bahnstationen Sternschanze und St. Pauli,

- das Elbufer entlang den Landungsbrücken,

- die Speicherstadt und

- das Areal vom Jungfernstieg über die Altstadt und Mönckebergstraße bis hin zum Hauptbahnhof.

Nachdem die tagestouristischen Aktionsräume und Hotspots bestimmt sind, wird nachfolgend analysiert, wo im Stadtraum Menschen verstärkt wahrgenommen werden.

\subsection{Wahrnehmung von Crowding}

Nachfolgend werden die empirischen Befunde über die Wahrnehmung der Tagestouristen in Bezug auf das Empfinden anderer Stadtnutzer dargelegt. Die Wahrnehmung von Crowding wurde dabei mit drei Verfahren gemessen. Zum einen wurde in Anlehnung an Shelby et al. (1989) eine 9er-Likert-Skala verwendet, um auch feine Unterschiede in der Wahrnehmung des städtischen Crowdings zu messen. Hinzu wurden die Tagesausflügler konkret nach Orten im Stadtraum 
befragt, die ihrer Meinung nach zu viele Menschen aufwiesen. Schließlich wurde auf Basis der von Popp (2012) herausgearbeiteten Aspekte des positiven und negativen urbanen Crowdings die touristische Einschätzung mithilfe von Statements auf einer 5er-Likert-Skala gemessen.

Bei einem Mittelwert von 4.56 ( $\mathrm{SD}=2.26, \mathrm{n}=163)$ auf der 9er-Crowdingskala hatte mehr als jeder fünfte Tagesausflügler (Low-2-Box: $22 \%$ ) den Eindruck, dass die Stadt ganz und gar nicht überfüllt war. Im Vergleich zu Studien in Naturräumen ist dieser Wert sehr hoch, ${ }^{9}$ jedoch im urbanen Umfeld erwartungsgemäß. $8 \%$ der Tagesausflügler hingegen gaben an, dass sie die Stadt am Tag ihres Tagesausflugs als extrem überfüllt angesehen haben (Top-2-Box). Bei Betrachtung unterschiedlicher Variablen hinsichtlich der Wahrnehmung von Crowding ${ }^{10}$ zeigt die Varianzanalyse nur eine signifikant höhere Wahrnehmung von Crowding an Samstagen $(M=4.74, S D=2.22, F=4.72, p=.03)$. Das anhand der 9er-Skala abgefragte Crowdingempfinden korreliert darüber hinaus einzig signifikant mit dem Willkommenheitsgefühl als Tourist in Hamburg ( $\rho=.243, p=.002, n=160)$. Je höher die Wahrnehmung von Crowding in der Stadt, desto höher ist das Gefühl von Unwillkommenheit als Hamburger Tourist und vice versa. 55 \% der Tagesausflügler gaben an, am Tag ihres Ausflugs an Orten gewesen zu sein, bei denen ihrer Meinung nach zu viele Menschen waren. Die genannten Orte auf die offene Fragestellung zeigen eine räumliche Konzentration um die Bereiche des Hamburger Hauptbahnhofs, über die Mönckebergstraße, den Rathausmarkt hin zum Jungfernstieg als auch - neben mehreren Einzelnennungen - die Reeperbahn, die Landungsbrücken und das Areal um die Elbphilharmonie auf (Abb. 9 in Abschnitt 4.4).

Das Polaritätsprofil in Abbildung 7 zeigt, wie alle Tagesausflügler (blaue Linie) verschiedene Aspekte des positiven und negativen Crowdings in Hamburg wahrgenommen haben und welche Anpassungsstrategien sie nutzten. Darüber hinaus wurden die Tagesgäste gefiltert, die eine erhöhte Wahrnehmung von Crowding aufwiesen (rote Linie). Es handelt sich dabei um Personen, die auf der 9erSkala eine Crowdingwahrnehmung von 7 und mehr ankreuzten und angaben, am Tag des Tagesausflugs an Orten mit aus ihrer Sicht zu vielen Menschen gewesen zu sein (=vom Crowding Betroffene). Dies trifft auf $22 \%$ der Tagesausflügler zu.

Zunächst fällt bei Betrachtung aller Tagesgäste auf, dass die Aspekte des positiven Crowdings im Vergleich zu den negativen Begleiterscheinungen deutlich überwiegen. Die höchsten Zustimmungswerte werden bei der Freude, unter-

9 So bspw. bei der Wahrnehmung von Crowding auf der Hamburger Hallig: $M=1.87, S D=1.61$, $\mathrm{n}=509$ (Kalisch, 2012, S. 74).

10 Aktivitäten, Reiseerfahrung, verbrachte Zeit in Hamburg, Geschlecht, Gruppengröße 


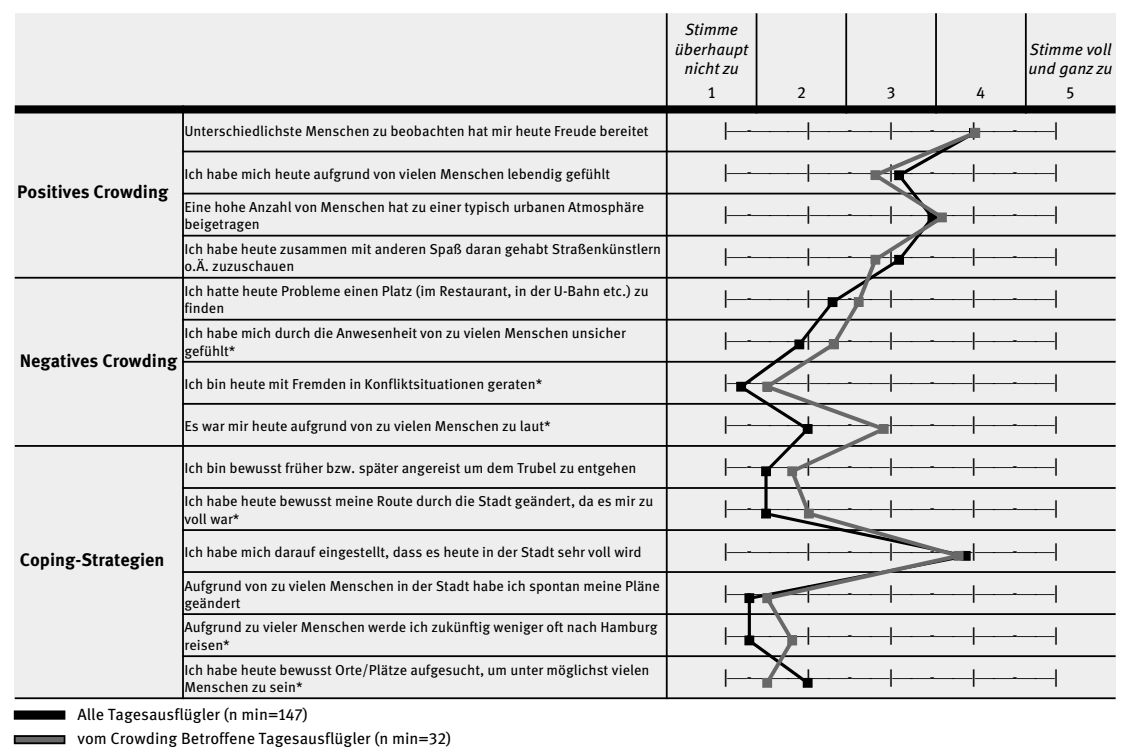

Abb. 7: Wahrnehmung von Crowding und Coping-Strategien von Hamburger Tagesausflüglern. Fragestellung: Wenn Sie an Ihren heutigen Tagesausflug denken, wie sehr können Sie den folgenden Aussagen zustimmen? Sie können Ihre Antworten auf einer Skala von 1=,stimme überhaupt nicht zu“ bis $5=$,stimme voll und ganz zu“ abstufen.)

* mindestens signifikant auf dem $5 \%$-Level ( $p \leq .05)$. Quelle: Eigene Erhebung, Berechnung und Darstellung.

schiedlichste Menschen $\mathrm{zu}$ beobachten gemacht ( $\mathrm{M}=4.03 ; \mathrm{SD}=1.09)$ sowie dass viele Menschen zu einer typisch urbanen Atmosphäre beigetragen haben $(M=3.49$; $\mathrm{SD}=1.33$ ). Die negativen Aspekte des Crowdings spielen fast keine Rolle im Hamburger Tagestourismus. So ist lediglich das Platzproblem mit einem leicht erhöhten Mittelwert von 2.26 (SD=1.39) als zu beobachtender Störfaktor zu identifizieren. Bei Betrachtung der Werte der vom Crowding betroffenen Tagestouristen werden zwei Punkte deutlich: Zum einen die in Summe erwartungsgemäß leicht niedrigeren Werte bei den positiven und entsprechend höheren Werte bei den negativen Aspekten, aber auch die Tatsache, dass die positive Wahrnehmung von Crowding selbst in dieser Gruppe (deutlich) überwiegt. Anders ausgedrückt: Einerseits nehmen sie die Stadt als überfüllt wahr, andererseits trägt die Anzahl vieler Menschen aber zu einer typisch urbanen Atmosphäre bei, bei der es auch Spaß macht, andere Menschen zu beobachten. Ähnlich verhält es sich bei den Unterschieden der beiden Gruppen hinsichtlich der Gesamtzufriedenheit mit dem Aufenthalt und dem Willkommenheitsgefühl als Tourist in der Stadt. Zwar sind die Werte der vom Crowding Betroffenen signifikant schlechter, gleichwohl kann auch in diesem Falle nicht davon gesprochen werden, dass diese Gruppe den Aufenthalt negativ bewertet oder sich gar als Tourist in Hamburg nicht willkommen fühlt (Tab. 2). 
Tab. 2: Unterschiede in der Gesamtzufriedenheit und im Willkommenheitsgefühl als Tourist bei Hamburger Tagesausflüglern. Fragestellung Zufriedenheit: Wie bewerten Sie alles in allem Ihren Aufenthalt heute in Hamburg? 1=sehr gut bis $5=$ mangelhaft. Fragestellung Willkommenheitsgefühl: Wie bewerten Sie alles in allem Ihren Aufenthalt heute in Hamburg? 1=sehr willkommen bis 5=unwillkommen. Quelle: Eigene Erhebung, Berechnung und Darstellung

\begin{tabular}{lccccc} 
Variable & \multicolumn{3}{c}{ Anova } \\
\hline Zufriedenheit mit Aufenthalt & $\mathrm{n}$ & $\mathrm{M}$ & $\mathrm{SD}$ & $\mathrm{F}$ & $\mathrm{p}$ \\
\hline vom Crowding Betroffene & & & & & \\
nicht vom Crowding Betroffene & 125 & 1,62 & 0,68 & & \\
Gesamt & 160 & 1,68 & 0,71 & & \\
Willkommenheitsgefühl als & & & & & \\
Tourist in Hamburg & & & & & \\
\hline vom Crowding Betroffene & 35 & 2,00 & 0,94 & 5,17 & 0,02 \\
nicht vom Crowding Betroffene & 128 & 1,67 & 0,70 & & \\
Gesamt & 163 & 1,74 & 0,77 & &
\end{tabular}

Hinsichtlich der Anpassungsstrategien zeichnet sich ein eindeutiges Bild einer Rationalisierungsstrategie für den Fall des Hamburger Tagestourismus ab. Demnach spielt die kognitive Strategie der Rationalisierung und die Vergegenwärtigung der Möglichkeit einer überfüllten Stadt im Vorfeld des Tagesausfluges die größte Rolle (65\% stimmen eher bzw. voll und ganz zu). Verhaltensorientierte Anpassungsstrategien, wie bspw. eine zeitliche oder räumliche Verschiebung des Aufenthaltes, haben sowohl im Gesamtsample als auch bei der Gruppe der Crowding Betroffenen eine untergeordnete Bedeutung inne. Die in Anlehnung an Namberger (2015) entwickelten und in Abstimmung mit der Praxis in Hamburg validierten und abgeänderten Items zur Wahrnehmung von tourismusbezogenen Phänomenen im Hamburger Stadttraum, zeigen wenige und insbesondere kaum handlungsauslösende - im Sinne einer Veränderung des Aktionsraumes - Störfaktoren auf. Lediglich bei zwei Phänomenen zeigt sich eine hohe Betroffenheit: betrunkene Touristen und Menschenmassen in Einkaufsstraßen (jeweils über 50 \% Wahrnehmung und Störung) (Abb. 8). Während im ersten Fall etwas mehr als ein Drittel der Tagesausflügler versuchen, dem Phänomen aus dem Weg zu gehen, werden die Menschenmassen in Einkaufsstraßen störend in Kauf genommen. So ist es zum einen das Verhalten der Menschen, welches als störend empfunden wird (Betrunkene, Fußballfans) und zum anderen die physische Enge. Die Befunde decken sich weitestgehend mit Ergebnissen aus München (Namberger, 2015; Kagermeier \& Erdmenger, 2019), obwohl hier Touristen und Bewohner zusammen betrachtet und ausgewertet wurden. 


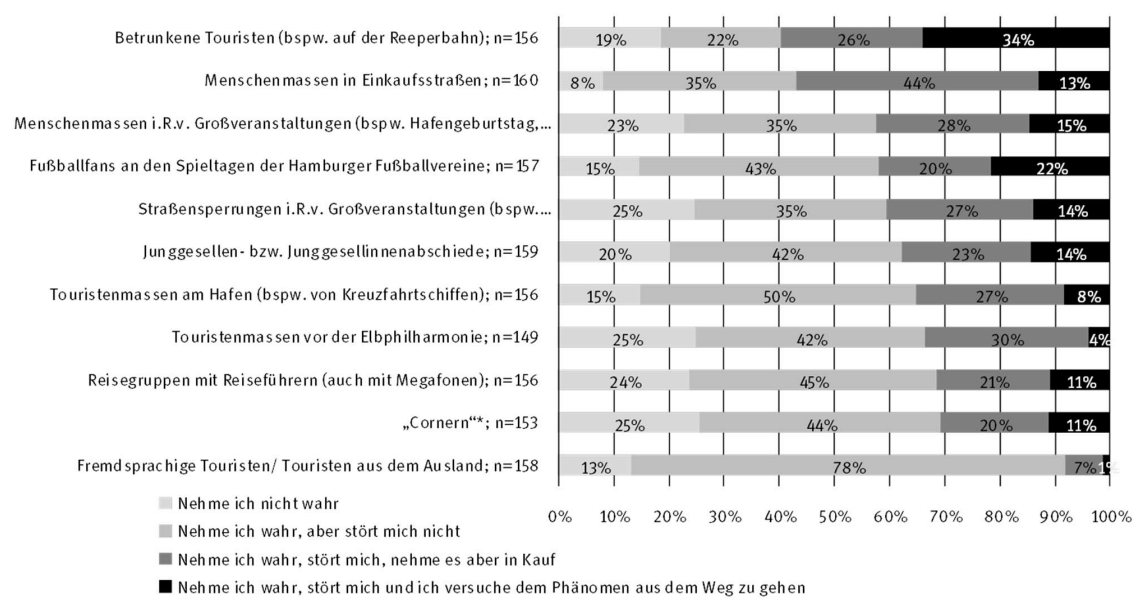

Abb. 8: Wahrnehmung touristischer Phänomene in Hamburg von Tagesausflüglern. Fragestellung: Folgende Phänomene treten in Hamburg innerhalb der Stadt auf. Nehmen Sie diese Phänomene grundsätzlich wahr? Falls ja, inwiefern stören Sie sich daran und inwieweit meiden Sie solche Orte, falls Sie sich daran stören? * (gemeinsames Beisammensein und Trinken) vor Kiosken/Eckkneipen an Sommerabenden. Quelle: Eigene Erhebung, Berechnung und Darstellung

\subsection{Synthese: Touristische Aktionsräume und Crowding}

In der Zusammenschau ermittelter Aktionsräume und Crowdingwahrnehmung lassen sich nun die Areale in Hamburg identifizieren, die als besonders anfällig für eine Verminderung der psychischen Tragfähigkeit angesehen werden können. Werden nach oben beschriebener Methodik lediglich die GPS-Trackpoints berücksichtigt, die von vom Crowding betroffenen Tagesausflüglern stammen, so zeigen sich mit wenigen Ausnahmen die identischen Hotspots (Abb. 9). Auch die Crowdingbetroffenen bewegen sich entlang der gleichen Routen durch Hamburg, was angesichts des subjektiven Prozesses der Wahrnehmung von Crowding nicht verwunderlich ist. Dies zeigt erneut auf, dass die Anwesenheit von vielen Menschen im Stadtraum zwar eine notwendige Bedingung für die Wahrnehmung von Crowding ist, die individuelle Wahrnehmung, Interpretation und Anpassungsstrategien jedoch stark subjektiv sind. Bei der Gegenüberstellung der derart ermittelten statistisch signifikanten Hotspots des Hamburger Tagestourismus von Crowding Betroffenen mit den Orten, an denen im Rahmen des Tagesausflugs $\mathrm{zu}$ viele Menschen wahrgenommen wurden, fällt die Lagegleichheit ins Auge. Es können somit zwei Kernzonen in Hamburg identifiziert werden, die in Bezug auf die Wahrnehmung von Crowding im Rahmen einer sozialverträglichen Tourismus- und Stadtentwicklung bevorzugt behandelt werden sollten: den Bereich um den Hauptbahnhof über die Spitaler- und Mönckebergstraße, den Rathausplatz 
hin zum Jungfernstieg sowie das Areal von den Landungsbrücken hin zur Elbphilharmonie (Abb. 9).

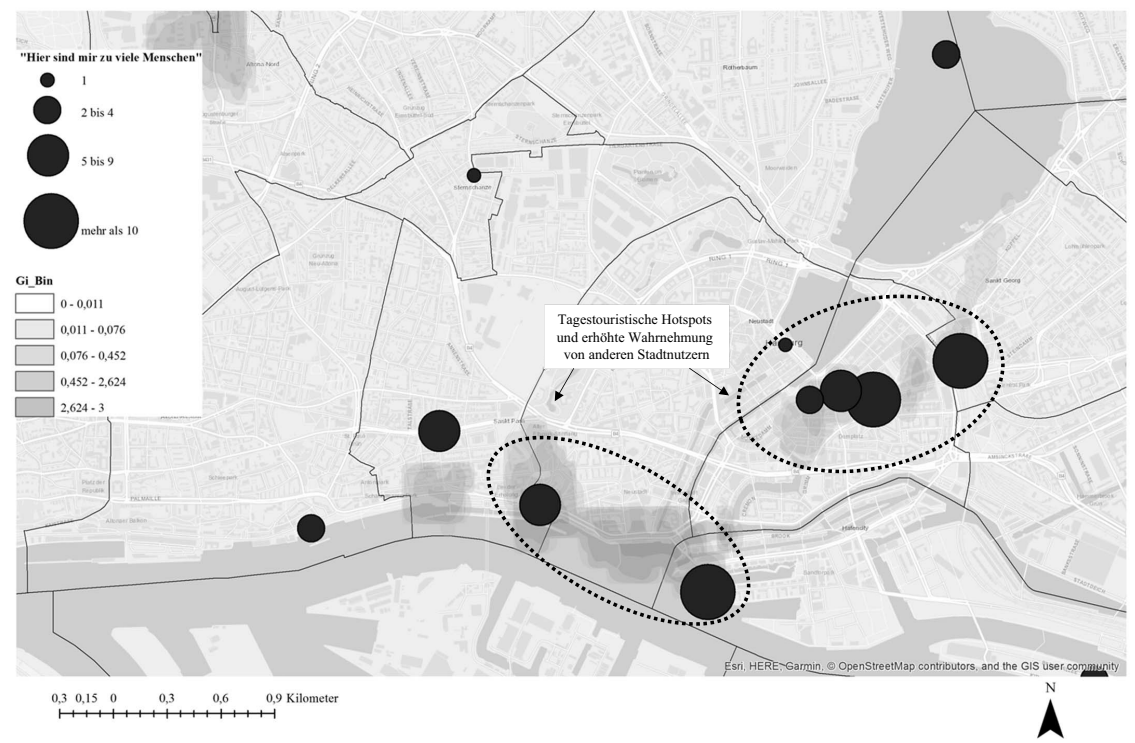

Abb. 9: Synthese von statistisch signifikanten Hotspots des Tagestourismus in Hamburg von Crowdingbetroffenen und räumliche Verteilung der Orte in Hamburg, bei denen alle Tagesausflügler zu viele Menschen wahrgenommen haben (Kartenausschnitt). $n=29$ GPS-Tracks von Crowdingbetroffenen. Räumliche Verteilung: Anzahl der Nennungen. Fragestellung: Sind Sie heute an Orten in Hamburg gewesen, von denen Sie sagen würden: „Hier sind mir zu viele Menschen?" Falls ja, welche Orte waren das (offene Nennung möglich). $n=117$ Nennungen. Quelle: Eigene Erhebung, Berechnung und Darstellung. Kartengrundlage: ALKIS Hamburg, ArcGis und OpenStreetMap

\section{$5 \quad$ Fazit und Ausblick}

Anhand der Ergebnisse konnte gezeigt werden, dass sich der private Tagestourismus in Hamburg auf wenige Hotspots des innerstädtischen Zentrums (CBD) konzentriert. Dies hängt $u$. a. damit zusammen, dass Tagesausflügler den Hauptaktivitäten Restaurantbesuch, Shopping, spazieren gehen und Sightseeing nachgehen und die zur Bedürfnisbefriedigung des Aufenthalts notwendige touristische Infrastruktur sich im innerstädtischen Zentrum befindet. Nur vereinzelt werden Stadtviertel außerhalb des Zentrums aufgesucht. Viele andere Stadtnutzer werden im Hamburger Städtetourismus seitens der Tagestouristen zwar wahrgenommen, das positive Crowding, allen voran die Freude, unterschiedliche Menschen zu beobachten, dominiert jedoch deutlich. $\mathrm{Zu}$ viele Menschen werden 
nur von wenigen Tagesausflüglern als störend empfunden, sie haben aber nahezu keinen Einfluss auf das aktionsräumliche Handeln vor Ort. So zeigt sich auch, dass es keine Unterschiede in den Aktionsräumen von Personen gibt, die eine erhöhte Wahrnehmung von Crowding aufweisen. Es konnten jedoch zwei Areale in Hamburg identifiziert werden, in denen es vonseiten des Destinationsmanagements zukünftig gilt, auf eine raumzeitliche Entzerrung und Besucherlenkung im Sinne einer sozialverträglichen Stadt- und Tourismusentwicklung zu setzen.

Um zukünftig den Abgleich von Aktionsräumen und der Wahrnehmung von (positivem) Crowding in Städten besser zu erforschen, bietet sich die Kombination von qualitativen in-situ-Methoden während des touristischen Erlebnisses und GPS-Tracking an. Der Vorteil der Nutzung qualitativer Methodik zeigt sich in einem tiefer gehenden Erkenntnisgewinn durch das emische, von innen geschilderte, Verstehen des Touristenverhaltens (Reif et al., 2017, S. 403). Denkbar wäre auch eine Ermittlung des Stressniveaus mittels der Messung der Hautleitfähigkeit von Touristen in Verbindung mit GPS-Tracking (u. a. Shoval et al., 2018; Schlosser \& Zeile, 2018). So könnte in den oben identifizierten Hotspots des Hamburger Tagestourismus in unterschiedlichen Zeiträumen und bei unterschiedlicher Anzahl sowie Zusammensetzung von Menschen (bspw. bei Events), das Stressniveau von Touristen untersucht werden. Videoaufnahmen sowie nachträgliche Gedankenprotokolle oder Befragungen in-situ könnten zusätzlich Aufschluss über die Gründe des über einen Armbandsensor ermittelten Stressniveaus geben.

\section{Literatur}

Ahas, R., Aasa, A., Roose, A., Mark, Ü. \& Silm, S. (2008). Evaluating passive mobile positioning data for tourism surveys: An Estonian case study. In Tourism Management 29, 3. S. 469-486.

Batista e Silva, F., Marín Herrera, M. A., Rosina, K., Ribeiro Barranco, R., Freire, S. \& Schiavina, M. (2018). Analysing spatiotemporal patterns of tourism in Europe at high-resolution with conventional and big data sources: In Tourism Management 68, S. 101-115. DOI: 10.1016/j. tourman.2018.02.020

Bauder, M. (2012). Erfahrungen aus dem GPS-Tracking individueller Mobilität von Touristen - Konsequenzen für die Weiterentwicklung als neue humangeographische Methode. In Strobl, J., Blaschke, Th. \& Griesebner, G. (Hrsg.), Angewandte Geoinformatik 2012. Beiträge zum 24. AGIT-Symposium Salzburg. Berlin. S. 420-429.

Bauder, M. (2018). Dynamiken des Städtetourismus in Deutschland. Wachstumspfade der übernachtungsstärksten Großstädte. In Standort. Zeitschrift für Angewandte Geographie 42, 2. S. 105-110. DOI: http://dx.doi.org/10.1007/s00548-018-0535-z

Bauder, M. \& Freytag, T. (2015). Visitor Mobility in the city and the effects of travel preparation. In Tourism Geographies 17, 5, S. 682-700. DOI: 10.1080/14616688.2015.1053971.

Becker, Chr. (1982). Aktionsräumliches Verhalten von Urlaubern im Mittelgebirge. Trier. (=Materialien zur Fremdenverkehrsgeographie 9). 
Becker, L.-S., Mattes, A. Reif, J. Krüger, M. Eisenstein, B., Zeiner, M., Harrer, B. \& Sporer, M. (2018). Regionales Tourismus-Satellitenkonto Hamburg 2015. Die ökonomische Bedeutung der Tourismuswirtschaft in Hamburg. Heide. [pdf] Abgerufen von: http://www.imt-fhw.de/ fileadmin/Dateien_imt/Unterlagen_Projekte/IMT_Regionales_Tourismus-Satellitenkonto_ Hamburg_2019.pdf (Letzter Zugriff: 26.02.2019).

Beeco, J. A. \& Hallo, J. C. (2014). GPS Tracking of Visitor Use: Factors Influencing Visitor Spatial Behavior on a Complex Trail System. In Journal of Park and Recreation Administration 32, 2. S. 43-61.

Beeco, J. A., Hallo, J. C., English, W. R. \& Giumetti, G. W. (2013). The importance of spatial nested data in understanding the relationship between visitor use and landscape impacts. In Applied Geography 45. S. 147-157. DOI: 10.1016/j.apgeog.2013.09.001

Beyer, M., Günther, W., Schmücker, D. \& Seidel, A. (2017). Nachhaltigkeitskonzeption Städtetourismus Hamburg. Kiel/Berlin.

Bryon, J. \& Neuts, B. (2008). Crowding and the Tourist Experience in an Urban Environment: $A$ Structural Equation Modeling Approach. [online] Abgerufen von: http://www.steunpunttoerisme.be/main/files/nieuwsbrief/oktober_2008/paperNVVS_bart_neuts.pdf (Letzter Zugriff: 30.12.2018).

Chatel-Messer, A. (2013). Heritage Interpretation als Element eines nachhaltigen Tourismus im Pilotprojekt Interpretationsraum Kandel, Südschwarzwald - eine Evaluation mittels GPS-Tracking. Dissertation. Freiburg im Breisgau.

Debbage, K.G. (1991). Spatial behavior in a bahamian resort. In Annals of Tourism Research 18, 2. S. 251-268. DOI: 10.1016/0160-7383(91)90008-Y

Edwards, D. \& Griffin, T. (2013). Understanding tourists' spatial behaviour. GPS tracking as an aid to sustainable destination management. In Journal of Sustainable Tourism 21, 4. S. 580-595.

Edwards, D., Dickson, T., Griffin, T. \& Hayllar, B. (2010). Tracking the Urban Visitor: Methods for Examining Tourists' Spatial Behaviour and Visual Representations. In Richards, G. und Munsters, W. (Hrsg.), Cultural tourism research methods. S. 104-114.

Eisenstein, B. (2014). Grundlagen des Destinationsmanagements. München.

Eisenstein, B., Reif, J., Krüger, J., Schmücker, D. \& Weis, R. (2019). Geschäftsreisen. Konstanz. (im Erscheinen).

ESRI (Hrsg.) (2018). Optimierte Hot-Spot-Analyse. [online] Abgerufen von: http://desktop.arcgis. $\mathrm{com} / \mathrm{de} / \mathrm{arcmap} / 10.3 /$ tools/spatial-statistics-toolbox/optimized-hot-spot-analysis.htm (Letzter Zugriff: 30.12.2018).

Eusébio, C., Vieira, A.L. \& Lima, S. (2018). Place attachment, host-tourist interactions, and residents' attitudes towards tourism development: The case of Boa Vista Island in Cape Verde. In Journal of Sustainable Tourism 26, 6. S. 890-909. DOI: 10.1080/09669582.2018.1425695

Flognfeldt jr., T. (2005). The tourist route system - models of travelling patterns. In belgeo 1-2, S. 35-58. DOI: 10.4000/belgeo.12406.

Freytag, T. (2010a). Visitor Activities and Inner-City Tourist Mobility: The Case of Heidelberg. In Mazanec, J. A. \& Wöber, K.W. (Hrsg.), Analysing International City Tourism. Wien. S. 213-226.

Freytag, T. (2010b). Déjà-vu Tourist practices of repeat visitors in the city of Paris. In Soc. Geogr. 5, 1. S. 49-58.

Freytag, T. \& Bauder M. (2018). Bottom-up touristification and urban transformations in Paris. In Tourism Geographies 20, 3. S. 443-460DOI: 10.1080/14616688.2018.1454504

Freytag, T. \& Gomes de Matos, C. (2018). Eine Reflektion über Grenzen der Touristifizierung. New Urban Tourism in Barcelona. Unvollständige Kurzfassung. Präsentation im Rahmen der 22. 
Jahrestagung der Deutschen Gesellschaft für Tourismuswissenschaft e.V. am 09.11.2019 an der FH Westküste.

Füller, H. \& Michel, B. (2014). 'Stop Being a Tourist!' New Dynamics of Urban Tourism in BerlinKreuzberg. In International Journal of Urban and Regional Research 38, 4. S. 1304-1318.

Girardin, F., Vaccari, A., Gerber, A., Biderman, A. \& Ratti, C. (2009). Quantifying urban attractiveness from the distribution and density of digital footprints. In International Journal of Spatial Data Infrastructures Research 4, S. 175-200.

Grube, N. \&Novy, J. (2018). Streitgrund Stadttourismus. Über die Ursachen und Folgen der Debatte über Overtourism und Tourismusphobie. In PLANERIN 6, 18. S. 5-8.

Hägerstrand, T. (1970). What about people in regional science? In Papers in Regional Science 24. S. 7-24.

Hamburg Tourismus GmbH (HHT) \& dwif-Consulting GmbH (Dwif) (Hrsg.) (2015). Wirtschaftsfaktor Tourismus Hamburg und die Metropolregion. [pdf] Abgerufen von: https://static1.hamburg-tourism.de/live_fileadmin/redaktion/Monitoring/HHT_ WirtschaftsfaktorTourismus_2014_web.pdf (Letzter Zugriff: 7.5.2017).

Jin, Q. \& Pearce, P. (2011). Tourist Perception of Crowding and Management Approaches at Tourism Sites in Xi'an'. In Asia Pacific Journal of Tourism Research 16, 3. S. 325-338. DOI: 10.1080/10941665.2011.572667

Judd, D. R. (1999). Constructing the tourist bubble. In Judd, D. R. \& Fainstein, S. S. (Hrsg.), The Tourist City. New Haven/London. S. 35-53.

Kagermeier, A. (2015). Tourismusgeographie. Konstanz/München.

Kagermeier, A. \& Erdmenger, E. (2019). Das Phänomen Overtourism: Erkundungen am Eisberg unterhalb der Wasseroberfläche. In Reif, J. \& Eisenstein, B., Tourismus und Gesellschaft: Kontakte - Konflikte - Konzepte. o. S. (im Erscheinen).

Kalisch, D. (2012). Recreational use of protected areas in Germany: Evaluation vistors“ perception of crowding in the Wadden Sea National Park. Dissertation. Berlin.

Kellner, L. \& Egger, R. (2016). Tracking Tourist Spatial-Temporal Behavior in Urban Places. A Methodological Overview and GPS Case Study. In Inversini, A. \& Schegg, R. (Hrsg.), Information and Communication Technologies in Tourism 2016. S. 481-494.

Keul, A. G. \& Kühberger, A. (1996). Die Strasse der Ameisen. Beobachtungen und Interviews zum Salzburger Städtetourismus. München. (=Tourismuswissenschaftliche Manuskripte 1).

Koens, K., Postma, A. \& Papp, B. (2018). Is Overtourism Overused? Understanding the Impact of Tourism in a City Context. In Sustainability 10,12. S. 1-15. Doi: 10.3390/su10124384

Lau, G. \& McKercher, B. (2006). Understanding tourist movement patterns in a destination: A GIS approach. In Tourism and Hospitality Research 7, 1. S. 39-49. DOI: 10.1057/palgrave. thr.6050027

Leiper, N. (1979). The Framework of Tourism. Towards a Definition of Tourism, Tourist and the Tourism Industry. In Annals of Tourism Research 6, 4. S. 390-407.

Leiper, N. (1990). Tourist attraction systems. In Annals of Tourism Research 17, 3. S. 367-384. DOI: 10.1016/0160-7383(90)90004-B.

Leung, X.Y., Wang, F., Wu, B.; Bai, B.; Stahura, K.A. \& Xie, Z. (2012). A Social Network Analysis of Overseas Tourist Movement Patterns in Beijing: the Impact of the Olympic Games. In International Journal of Tourism Research 14, 5. S. 469-484. DOI: 10.1002/jtr.876

Lew, A. \& McKercher, B. (2006). Modeling Tourist Movements. In Annals of Tourism Research 33, 2. S. 403-423. DOI: 10.1016/j.annals.2005.12.002. 
Lorkowski, A. (2017). Einwohnerbefragung zur Akzeptanz des Tourismus. Repräsentative Bürgerbefragung 2016. Präsentation im Rahmen des Treffens der LMO-Marktforscher am 01.06.2017 an der FH Westküste.

Maitland, R. \& Newman, P. (2004). Developing metropolitan tourism on the fringe of central London. In International Journal of Tourism Research 6, 5. S. 339-348. DOI: 10.1002/jtr.496

Martin, B.S. \& Uysal, M. (1990). An examination of the relationship between carrying capacity and the tourism lifecycle: Management and policy implications. In Journal of Environmental Management 31, 4. S. 327-333. DOI: 10.1016/S0301-4797(05)80061-1

Maschke, J. (2014). Sonderauswertung der Grundlagenstudie „Tagesreisen der Deutschen“ für die Stadt und die übrige Metropolregion. (unveröffentliches Dokument per Mail am 22.11.2018)

Massey, D. (2005). For Space. London.

McCool, S.F. \& Lime, D.W. (2001). Tourism Carrying Capacity: Tempting Fantasy or Useful Reality? In Journal of Sustainable Tourism 9, 5. S. 372-388. DOI: $10.1080 / 09669580108667409$

McKercher, B. (2004). The myth of the average tourist. In Voice of TIC 4. S. 19-24.

McKercher, B. \& Lau, G. (2008). Movement Patterns of Tourists within a Destination. In Tourism Geographies 10, 3. S. 355-374. DOI: 10.1080/14616680802236352

McKercher, B. \& Lau, G. (2009). Methodological Considerations when mapping tourist movements in a destination. In Tourism Analysis 14, 4. S. 443-455. DOI: 10.3727/1083542 09X12596287114138

McKinsey \& Company \& World Travel \& Tourism Council (WTTC) (Hrsg.) (2017). Coping With Success: Managing Overcrowding in Tourism Destinations 2017. o.0.

Muler Gonzalez, V., Coromina, L. \& Galí, N. (2018). Overtourism: residents' perceptions of tourism impact as an indicator of resident social carrying capacity - case study of a Spanish heritage town. In Tourism Review 73, 3. S. 277-296. DOI: https://doi.org/10.1108/ TR-08-2017-0138

Namberger, Ph. (2015). Touristische Übernutzung Münchens: Wie viel Tourismus verträgt die bayerische Landeshauptstadt? Vortrag auf dem Deutschem Kongress für Geographie, Berlin, 1. bis 6. Oktober 2015.

Neuts, B. \& Nijkamp, P. (2012). Tourist Crowding Perception and acceptability in cities. An Applied Modelling Study on Bruges. In Annals of Tourism Research 33, 4. S. 2133-2153. DOI: http://dx.doi.org/10.1016/j.annals.2012.07.016

Neuts, B. \& Vanneste, D. (2018). Contextual Effects on Crowding Perception: An Analysis of Antwerp and Amsterdam. In Tijdschrift voor Economische en Sociale Geografie 109, 3. S. 402-419. DOI:10.1111/tesg.12284.

Neuts, B., Nijkamp, P. \& van Leeuwen, E. (2012). Crowding Externalities from Tourist Use of Urban Space. In Tourism Economics 18, 3. S. 649-670. DOI: 10.5367/te.2012.0130

Oppermann, M. (1995). A Model of Travel Itineraries. In Journal of Travel Research 33, 4. S. 57-61. Osterhage, F. \& Wiegandt, C.-C. (2014). Wohnstandorte und Aktionsräume: Leben zwischen Quartier und Region. In Danielzyk, R., Lentz, S. \& Wiegandt, C.-C. (Hrsg.), Suchst du noch oder wohnst du schon? Wohnen in polyzentrischen Stadtregionen. Berlin. S. 91-120.

Pappalepore, I. \& Smith, A. (2016). The Co-creation of urban tourism experiences. In Russo, A. \& Richards, G. (Hrsg.), Reinventing the local in tourism. Producing, consuming and negotiating place. Buffalo. S. 87-100. (=Aspects of tourism 73).

Pearce, P. (2011) Tourist behaviour and the contemporary world. Bristol. 
Peeters, P., Gössling, S., Klijs, J., Milano, C., Novelli, M., Dijkmans, C. ... \& Postma, A. (2018). Research for TRAN Committee - Overtourism: impact and possible policy responses.

Brüssel. [pdf] Abgerufen von: http://www.europarl.europa.eu/thinktank/en/document. html?reference=IPOL_STU(2018)629184. (Letzter Zugriff: 27.02.2019).

Pohl, Th. (2010). Chronomaps als Repräsentations- und Kommunikationsmittel der raum-zeitlichen Strukturierung. In Läpple, D., Mückenberger, U. \& Oßenbrügge, J. (Hrsg.), Zeiten und Räume der Stadt: Theorie und Praxis. Opladen. S. 159-181.

Popp, M. (2012). Positive and Negative Urban Tourist Crowding: Florence, Italy. In Tourism Geographies 14, 1. S. 50-72. DOI: 10.1080/14616688.2011.597421.

Popp, M. (2017). When walking is no longer possible. Investigating crowding and coping practices in urban tourism using commented walks. In Hall, C. M., Ram, Y. \& Shoval, N. (Hrsg.), The Routledge international handbook of walking. Abingdon, Oxon/New York S. 360-368.

Postma, A. \& Schmücker, D. (2017). Understanding and overcoming negative impacts of tourism in city destinations. Conceptual model and strategic framework. In Journal of Tourism Futures 3, 2. S. 144-156. DOI: 10.1108/JTF-04-2017-0022.

Reif, J. (2019). Die Nutzung von Mobilfunkdaten in der Tourismusforschung - Das Beispiel Tagestourismus in Hamburg. In Groß, S., Peters, J., Roth, R., Schmude, J. \& Zehrer, A. (Hrsg.), Wandel im Tourismus. Internationalität, Demografie und Digitalisierung. S. 31-50.

Reif, J., Hallerbach, B. \& May C. (2017): Qualitative Leitfadeninterviews als Ergänzung oder Alternative zu quantitativen Vor-Ort-Gästebefragungen - Ergebnisse des „Smart Focus“ aus der Cittaslow-Stadt Deidesheim. In Eisenstein, B. (Hrsg.), Marktforschung für Destinationen. Grundlagen - Instrumente - Praxisbeispiele. Berlin. S. 403-415.

Roseman, C.C. (1971). Migration as a spatial and temporal process. In Annals of the Association of American Geographers 61, 3), S. 589-598. DOI: 10.1111/j.1467-8306.1971.tb00809.x

Schamel, J. (2017). Raumzeitliches Verhalten bei der Ausübung landschaftsbezogener Erholungsaktivitäten. Dissertation. Würzburg.

Schamel, J. \& Job, H. (2014). Crowding in Germany's national parks: The case of the low mountain range Saxon Switzerland National Park. In eco.mont: Journal on Protected Mountain Areas Research 5, 1. S. 27-34. DOI: 10.1553/eco.mont-5-1s27

Scheiner, J. (1998). Aktionsraumforschung auf phänomenologischer und handlungstheoretischer Grundlage. In Geographische Zeitschrift 86, 1. S. 50-66.

Scheiner, J. (2000). Eine Stadt, zwei Alltagswelten? Ein Beitrag zur Aktionsraumforschung und Wahrnehmungsgeographie im vereinten Berlin. Berlin. (=Abhandlungen - Anthropogeographie 62).

Schlosser, F. \& Zeile, P. (2018). Angsträume und Stressempfinden im urbanen Kontext. In Schrenk, M., Popovich, V., Zeile, P., Elisei, P., Beyer, C \& Navratil, G. (Hrsg.) (2018). REAL CORP 2018. Proceedings/Tagungsband. Wien. S. 75-85.

Schmude, J. \& Namberger, Ph. (2015). Tourismusgeographie. Darmstadt.

Schüssler, N. \& Axhausen, K.W. (2008). Identifying trips and activities and their characteristics from GPS raw data without further information. In Arbeitsberichte Verkehrs- und Raumplanung 502. DOI: 10.3929/ETHZ-A-005589980

Shelby, B., Vaske, J. J. \& Heberlein, T.A. (1989). Comparative analysis of crowding in multiple locations: Results from fifteen years of research. In Leisure Sciences 11, 4. S. 269-291. DOI: $10.1080 / 01490408909512227$

Shoval, N. (2002). Spatial activity of tourists in cities: What are the underlying factors? In Wöber, K. W. (Hrsg.); City Tourism 2002. Wien und New York. S. 18-33. 
Shoval, N. \& Ahas, R. (2016). The use of tracking technologies in tourism research: the first decade. In Tourism Geographies 18, 5. S. 587-606.

Shoval, N. \& Isaacson, M. (2010). Tourist Mobility and Advanced Tracking Technologies. New York/London.

Shoval, N., McKercher, B., Ng, E. \& Birenboim, A. (2011). Hotel location and tourist activity in cities. In Annals of tourism research 38, 4. S. 1594-1612.

Shoval, N., Schvimer, Y. \& Tamir, M. (2018). Tracking technologies and urban analysis: Adding the emotional dimension. In Cities 72. S. 34-42. DOI: 10.1016/j.cities.2017.08.005

Statistisches Bundesamt (Hrsg.) (2008). Tourismus in Zahlen 2007. Wiesbaden.

Statistisches Bundesamt (Hrsg.) (2018). Tourismus in Zahlen 2017. Wiesbaden.

Steinbach, J. (2003). Tourismus. Einführung in das räumlich-zeitliche System. München.

Stokols, D. (1972). On the distinction between density and crowding: Some implications for future research. In Psychological Review 79, 3. S. 275-277. DOI: $10.1037 / \mathrm{h} 0032706$

Stors, N. \& Kagermeier, A. (2017). The sharing economy and its role in metropolitan tourism. In Gravari-Barbas, M. \& Guinand, S. (Hrsg.) (2017). Tourism and Gentrification in Contemporary Metropolises: International Perspectives. Milton. S. 181-206.

Sun, Y.-Y. \& Budruk, M. (2017). The moderating effect of nationality on crowding perception, its antecedents, and coping behaviours: A study of an urban heritage site in Taiwan. In Current Issues in Tourism 20, 12. S. 1246-1264. DOI: 10.1080/13683500.2015.1089845

United Nations Department of Economic and Social Affairs, Statistics Division (UNSD) und United Nations World Tourism Organization (UNWTO) (Hrsg.) (2010). International Recommendations for Tourism Statistics 2008. New York.

Urry, J. (2002). The Tourist Gaze. Second Edition. London.

Vaske, J. J. \& Shelby, B. (2008). Crowding as a Descriptive Indicator and an Evaluative Standard: Results from 30 Years of Research. In Leisure Sciences 30. S. 111-126. DOI: 10.1080/01490400701881341

Vester, H.-G. (1993). Crowding. In Hahn, H. \& Kagelmann, H. J. (Hrsg.); Tourismuspsychologie und Tourismussoziologie. Ein Handbuch zur Tourismuswissenschaft. München. S. 125-126.

Watson, G. L. \& Kopachevsky, J. P. (1996). Tourist Carrying Capacity: A Critical Look at the Discursive Dimension. In Progress in Tourism and Hospitality Research 2. S. 169-179.

Weber, H.-J. L. \& Bauder, M. (2013). Neue Methoden der Mobilitätsanalyse. Die Verbindung von GPS-Tracking mit quantitativen und qualitativen Methoden im Kontext des Tourismus. In Raumforschung und Raumordnung 71, 2. S. 99-113.

Wickham, T. \& Kerstetter, D. (2000). The relationship between place attachment and crowding in an event setting. In Event Management 6, 3. S. 167-174.

Wöhler, K. (2001). Tourismusmarketing. In Tscheulin, D.K. \& Helmig, B. (Hrsg.), Branchenspezifisches Marketing: Grundlagen - Besonderheiten - Gemeinsamkeiten. Wiesbaden. S. 187-202.

Wybraniec, J. (2018). „Tourist go home!“ - Entwicklung und Analyse von Regulierungsansätzen zur Vermeidung von Overtourism am Beispiel der Hansestadt Hamburg. Masterarbeit. Eberswalde.

Xiao-Ting, H. \& Bi-Hu, W. (2012). Intra-attraction Tourist Spatial-Temporal Behaviour Patterns. In Tourism Geographies 14, 4. S. 625-645. DOI: 10.1080/14616688.2012.647322

Zehrer, A. \& Raich, F. (2016). The impact of perceived crowding on customer satisfaction. In Journal of Hospitality and Tourism Management 29. S. 88-98. DOI: 10.1016/j. jhtm.2016.06.007 


\section{Autoreninformationen}

\section{Dipl.- Geogr. Julian Reif}

Institut für Management und Tourismus (IMT), Fachhochschule Westküste, Fritz-ThiedemannRing 20, D-25746 Heide, reif@fh-westkueste.de

Julian Reif, Dipl.-Geogr., studierte an den Universitäten Bonn und Fribourg Geographie mit den Nebenfächern Soziologie und Ethnologie. Seit 2012 ist er wissenschaftlicher Mitarbeiter und Projektleiter im Institut für Management und Tourismus (IMT) der FH Westküste und Referent der Institutsleitung. Von 2012 bis 2015 war er an der FH Westküste zudem Dozent u. a. für Destinationsmanagement, Tourismusmarktforschung und Methodenlehre. Zuvor war er von 2009 bis 2011 als Travel Consultant bei der moveo Studienreisen GmbH tätig. Seine Forschungsinteressen sind touristische Nachfragetrends, Städtetourismus, Auswirkungen des Tourismus und aktionsräumliches Verhalten. Zurzeit promoviert er in der Arbeitsgruppe Stadt- und Regionalforschung bei Prof. Dr. Claus-Christian Wiegandt im Geographischen Institut der Rheinischen Friedrich-Wilhelms-Universität Bonn über Aktionsräume von Touristen in Städten. 
